\title{
Operators with an absolute functional calculus
}

\author{
N. J. Kalton • T. Kucherenko
}

Received: 4 December 2008 / Revised: 8 May 2009 / Published online: 21 August 2009

(C) The Author(s) 2009. This article is published with open access at Springerlink.com

\begin{abstract}
We study sectorial operators with a special type of functional calculus, which we term an absolute functional calculus. A typical example of such an operator is an invertible operator $A$ (defined on a Banach space $X$ ) considered on the real interpolation space $(\operatorname{Dom}(A), X)_{\theta, p}$ where $0<\theta<1$ and $1<p<\infty$. In general the absolute functional calculus can be characterized in terms of real interpolation spaces. We show that operators of this type have a strong form of the $H^{\infty}$-calculus and behave very well with respect to the joint functional calculus. We give applications of these results to recent work of Arendt, Batty and $\mathrm{Bu}$ on the existence of Hölder-continuous solutions for the abstract Cauchy problem.
\end{abstract}

\section{Introduction}

Let $A$ be a sectorial operator on a complex Banach space $X$ with domain $\operatorname{Dom}(A)$. It is a well-established principle that the properties of $A$ improve when $A$ is considered as an operator on the real interpolation spaces $(\operatorname{Dom}(A), X)_{\theta, p}$ where $0<\theta<1$ and $1 \leq p \leq \infty$. Examples of such properties are $H^{\infty}$-calculus and joint functional calculus, which are defined below. This idea goes back to Berens and Butzer [4] and Da Prato and Grisvard [13] (see also Lunardi [21]). A typical recent result is that of Dore [14] who shows that if $A$ is invertible then $A$ has an $H^{\infty}$-calculus on the

\footnotetext{
N. J. Kalton

Department of Mathematics, University of Missouri-Columbia, Columbia, MO 65211, USA

e-mail: nigel@math.missouri.edu

T. Kucherenko $(\varangle)$

Department of Mathematics, University of California-Los Angeles, Los Angeles, CA 90095, USA

e-mail: tamara@math.ucla.edu
} 
interpolation space $(\operatorname{Dom}(A), X)_{\theta, p}$ where $1 \leq p<\infty$ and $0<\theta<1$. See also [15] for the non-invertible case, where $\operatorname{Dom}(A)$ is replaced by $\operatorname{Dom}(A) \cap \operatorname{Ran}(A)$.

Let us recall that a sectorial operator $A$ of type $\omega=\omega(A)$ on a complex Banach space $X$ is a closed one-one operator with dense domain and range satisfying an estimate

$$
\|\lambda R(\lambda, A)\| \leq C_{\phi}, \quad|\arg \lambda| \geq \phi
$$

whenever $\phi>\omega$. If $f \in H^{\infty}\left(\Sigma_{\phi}\right)$ is bounded and analytic on the sector $\Sigma_{\phi}=\{z \in$ $\mathbb{C} \backslash\{0\}:|\arg z|<\phi\}$ for some $\phi>\omega$ we can define $f(A)$ as a densely defined closed operator. We say that $A$ has an $H^{\infty}\left(\Sigma_{\phi}\right)$-calculus if for every $f \in H^{\infty}\left(\Sigma_{\phi}\right), f(A)$ extends to a bounded operator and we have an estimate

$$
\|f(A)\| \leq C\|f\|_{H^{\infty}\left(\Sigma_{\phi}\right)}
$$

We then let $\omega_{H}(A)$ be the infimum of all such $\phi$ (in general $\omega_{H}(A) \geq \omega(A)$ ).

It is an important observation that the theory of sectorial operators on a Hilbert space is, in general, simpler and more easily applicable than in general Banach spaces. This is mainly due to a characterization of Hilbert spaces as certain interpolation spaces related to an operator with $H^{\infty}$-calculus. Therefore, let us focus on sectorial operators with $H^{\infty}$-calculus on a Banach space. Such operators were first studied by McIntosh in the special setting of Hilbert spaces [22]. Let $\mathcal{D}(A)$ denote the completion of $\operatorname{Dom}(A)$ under the norm $x \rightarrow\|A x\|$ and let $\mathcal{R}(A)=\mathcal{D}\left(A^{-1}\right)$. In [3], it was shown that a sectorial operator $A$ on a Hilbert space has an $H^{\infty}$-calculus if and only if $X$ can be identified with the complex interpolation space $[\mathcal{D}(A), \mathcal{R}(A)]_{1 / 2}$, which coincides with the real interpolation space $(\mathcal{D}(A), \mathcal{R}(A))_{1 / 2,2}$, These results are proved using certain quadratic estimates, for which there is no analogue in a general Banach space.

As another example, consider the joint functional calculus developed in [18]. Suppose $(A, B)$ are two commuting sectorial operators and that $A$ has an $H^{\infty}$-calculus. If $\phi_{A}>\omega_{H}(A)$ and $\phi_{B}>\omega(B)$ then for $f \in H^{\infty}\left(\Sigma_{\phi_{A}} \times \Sigma_{\phi_{B}}\right)$ the operator $f(A, B)$ is bounded provided the collection of operators $\left\{f(w, B) ; w \in \Sigma_{\phi}\right\}$ is R-bounded (see [18] for the definition). R-boundedness may not be easy to verify in a concrete situation. Hence, it is important to know when a simpler condition suffices. If $X$ is a Hilbert space then requiring R-boundedness reduces to the much easier condition that

$$
\sup \left\{\|f(w, B)\| ; \quad w \in \Sigma_{\phi}\right\}<\infty .
$$

In [18] (Theorem 7.1), it is however shown that for certain other special Banach spaces the R-boundedness assumption can also be replaced by simple boundedness; this holds for example if $X=L_{1}$.

The aim of this paper is to provide a framework to understand the special properties of a sectorial operator $A$ which allow us to prove a joint functional calculus result with only a boundedness of the type (1.1); it turns out that this has a strong connection with real interpolation methods. We introduce the concept of an absolute functional calculus, which is significantly stronger than an $H^{\infty}$-calculus. 
Suppose $A$ is a sectorial operator of type $\omega$ and that $\phi>\omega$. We recall that $H_{0}^{\infty}\left(\Sigma_{\phi}\right)$ consists of all those functions $f \in H^{\infty}\left(\Sigma_{\phi}\right)$ which obey an estimate of the type $|f(z)| \leq C|z|^{\epsilon}(1+|z|)^{-2 \epsilon}$ for some $\epsilon>0$. Then $A$ is said to have an absolute functional calculus if there exists $\phi>\omega$ and $g, h \in H_{0}^{\infty}\left(\Sigma_{\phi}\right)$ so that for some constant $C$ we have the implication

$$
\|h(t A) g(t A) x\| \leq\|g(t A) y\|, \quad 0<t<\infty \Longrightarrow\|x\| \leq C\|y\|
$$

for $x, y \in X$. If $A$ has an absolute functional calculus then $A$ automatically has an $H^{\infty}$-calculus and a boundedness condition of type (1.1) becomes sufficient in the joint functional calculus (Theorems 4.2 and 6.4).

In practical situations, it is more natural to make an explicit choice for $g, h$ in (1.2). Let us write $\varphi_{a, b}(z)=z^{a}(1+z)^{-a-b}$ for $a, b>0$. We will say that $A$ has an $(a, b)$-absolute functional calculus if

$$
\left\|\varphi_{a+\delta, b+\delta}(t A) x\right\| \leq\left\|\varphi_{a, b}(t A) y\right\|, \quad 0<t<\infty \Longrightarrow\|x\| \leq C\|y\|
$$

This is a somewhat more restrictive concept (see Proposition 4.3 and the example following). However, this concept fits very naturally with interpolation theory. To make this more precise, let $\left(W_{0}, W_{1}\right)$ be a Banach couple; we say that an interpolation space $W$ is a strict real interpolation space if it is K-monotone of quasi power-type and regular (cf. [8] and Sect. 2, Proposition 2.3 and Theorem 2.4).

Let $A$ be a sectorial operator on the Banach space $X$. We show (Corollary 5.6) that if $Y$ is any strict real interpolation space for a couple $\left(\mathcal{D}\left(A^{\sigma}\right), \mathcal{D}\left(A^{\tau}\right)\right)$ where $\sigma<\tau$ then $A$ has a $(c, c)$-absolute functional calculus on $Y$ for some $c>0$. Then in Theorem 5.8, we show that if $X$ is a strict real interpolation space for the couple $\left(\mathcal{D}\left(A^{-b}\right), \mathcal{D}\left(A^{a}\right)\right)$ then $A$ has a $(c, c)$-absolute functional calculus for some $c<a+b$ while conversely if $A$ has an $(a, b)$-absolute functional calculus then $X$ is a strict real interpolation space for the couple $\left(\mathcal{D}\left(A^{-b^{\prime}}\right), \mathcal{D}\left(A^{a^{\prime}}\right)\right)$ for any $a^{\prime}>a$ and $b^{\prime}>b$. Thus having an $(a, b)$-absolute functional calculus (for some $a, b>0$ ) is roughly speaking a characteristic property of spaces obtained by strict real interpolation methods. Typically this applies when considering standard differential operators on a space of Besov type which are reached by real interpolation between Sobolev spaces.

In Sects. 7 and 8, we illustrate an application of these ideas by discussing the question whether the equation $A x+B x=y$ is well-posed when $A$ is a sectorial operator with an absolute functional calculus and $B$ is a closed operator which commutes with $A$. Our main goal for these sections is to extend and improve some results of Arendt et al. [2]. Let $\mathbb{T}$ denote the unit circle $(=[0,2 \pi))$ and suppose $0<\alpha<1$. Let $\mathcal{C}^{\alpha}(\mathbb{T} ; X)$ denote the space of $X$-valued $\alpha$-Hölder continuous functions on $\mathbb{T}$. Arendt et al. proved that if $B$ is a closed operator on $X$ with $\operatorname{Sp}(B) \cap i \mathbb{Z}=\emptyset$ satisfying the condition

$$
\left\|(i k+B)^{-1}\right\| \leq C k^{-2 / 3}
$$


then the equation

$$
u^{\prime}(t)+B u(t)=x(t), \quad u(0)=u(2 \pi)
$$

has a unique mild solution $u \in \mathcal{C}^{\alpha}(\mathbb{T} ; X)$ for every $x \in \mathcal{C}^{\alpha}(\mathbb{T} ; X)$. A special case of our results shows that the exponent $2 / 3$ can be improved to $1 / 2$; this was shown in [2] only under the additional assumption that $X$ has nontrivial type. The key reason for these results is the observation that the differentiation operator has an absolute functional calculus when considered on the space of functions of mean zero in the little Hölder space $\mathcal{C}_{0}^{\alpha, 0}(\mathbb{T} ; X)$. Our argument is based on the fact that $\mathcal{C}_{0}^{\alpha, 0}(\mathbb{T} ; X)$ can be identified with the interpolation space $\left(\mathcal{C}_{0}(\mathbb{T}, X), \mathcal{D}(A)\right)_{\alpha, \infty}$.

The results of this paper were announced in [17].

\section{Banach function spaces and interpolation spaces}

In this section, we review important facts about Banach function spaces and interpolation.

Consider the measure space $(0, \infty)$ with measure $d t / t$. Let $L_{0}(0, \infty)$ denote the space of all measurable functions on $(0, \infty)$ where functions coinciding almost everywhere are identified; let $\mathcal{C}_{00}(0, \infty)$ denote the subspace of essentially bounded functions of compact support in $(0, \infty)$. $L_{0}$ is a Hausdorff topological vector space under the topology of convergence in measure on sets of finite measure. We denote by $D_{s}: L_{0} \rightarrow L_{0}$ the linear map $D_{s} f(t)=f(t / s)$. Let $E$ be a linear subspace of $L_{0}$ equipped with a norm $\|\cdot\|_{E}$ so that $E$ is a Banach space. We shall say that $E$ is a Banach function space if the following conditions hold:

(i) $\mathcal{C}_{00}(0, \infty) \subset E$.

(ii) If $f \in E$ then $f$ is locally integrable.

(iii) If $f, g$ are measurable functions with $|f| \leq|g|$ a.e. and $g \in E$ then $f \in E$ and $\|f\|_{E} \leq\|g\|_{E}$.

(iv) The inclusion $E \hookrightarrow L_{0}$ is continuous.

We say $E$ is admissible if, in addition, we have

(v) For each $s \in(0, \infty)$ the operator $D_{s}: E \rightarrow E$ is bounded.

(vi) $\mathcal{C}_{00}(0, \infty)$ is dense in $E$.

Sometimes for convenience we will use the notation

$$
\|f\|_{E}=\|f(t)\|_{E, t}
$$

to indicate the variable.

For an admissible space $E$ the Boyd indices are defined as

$$
\begin{gathered}
\beta_{E}=\limsup _{s \rightarrow \infty} \frac{\log \left\|D_{s}\right\|}{\log s} \\
\alpha_{E}=\liminf _{s \rightarrow 0} \frac{\log \left\|D_{s}\right\|}{\log s}
\end{gathered}
$$


Lemma 2.1 Let $E$ be an admissible Banach function space. Then

1. $-\infty<\alpha_{E} \leq \beta_{E}<\infty$

2. If $a<\alpha_{E}$ and $b>\beta_{E}$ then there exists a constant $C=C(E, a, b)$ such that

$$
\left\|D_{s}\right\| \leq C \max \left(s^{a}, s^{b}\right)
$$

3. If $a<\alpha_{E}$ and $b>\beta_{E}$ then $\min \left(t^{-a}, t^{-b}\right) \in E$.

4. If $f \in E$ then $\lim _{r \rightarrow \infty}\left\|f \chi_{\left(0, r^{-1}\right)}+f \chi_{(r, \infty)}\right\|_{E}=0$.

5. If $f_{n} \in E$ are such that $\sup _{n}\left|f_{n}\right| \in E$ and $f_{n}(t)$ converges to $f(t)$ uniformly on compact subsets of $(0, \infty)$ then $\lim _{n \rightarrow \infty}\left\|f-f_{n}\right\|_{E}=0$.

Proof (1) and (2) are standard and we omit them. For (3) observe that $\chi_{[1,2]} \in E$ and hence so does $\sum_{n \in \mathbb{Z}} \min \left(2^{-n a}, 2^{-n b}\right) \chi_{\left[2^{n}, 2^{n+1}\right]}$.

(4): given $\epsilon>0$ we can pick $g \in \mathcal{C}_{00}$ with $\|f-g\|_{E}<\epsilon$. Now

$$
\limsup _{r \rightarrow \infty}\left\|f \chi_{\left(0, r^{-1}\right)}+f \chi_{(r, \infty)}\right\|_{E} \leq \epsilon+\limsup _{r \rightarrow \infty}\left\|g \chi_{\left(0, r^{-1}\right)}+g \chi_{(r, \infty)}\right\|_{E}=\epsilon .
$$

For (5) note that $|f| \leq g=\sup _{n}\left|f_{n}\right| \in E$ so that $f \in E$. Then for any $1<r<\infty$ we have using (iii) that

$$
\lim _{n \rightarrow \infty}\left\|\left(f-f_{n}\right) \chi_{\left[r^{-1}, r\right]}\right\|_{E}=0
$$

Choose $1<r_{n} \uparrow \infty$ so that

$$
\lim _{n \rightarrow \infty}\left\|\left(f-f_{n}\right) \chi_{\left[r_{n}^{-1}, r_{n}\right]}\right\|_{E}=0 .
$$

Then

$$
\left\|\left(f-f_{n}\right)-\left(f-f_{n}\right) \chi_{\left[r_{n}^{-1}, r_{n}\right]}\right\|_{E} \leq 2\left\|g-g \chi_{\left[r_{n}^{-1}, r_{n}\right]}\right\|_{E}
$$

and so using (4) we have $\lim _{n \rightarrow \infty}\left\|f-f_{n}\right\|_{E}=0$.

We now give a few examples to illustrate our setup.

(i) If $E=L_{p}(d t)$ we have $\left\|D_{s} f\right\|=\left(\int_{0}^{\infty}|f(t / s)|^{p} d t\right)^{1 / p}=s^{1 / p}\|f\|$ and so $\left\|D_{s}\right\|=s^{1 / p}$. Hence, $\alpha_{L_{p}}=\beta_{L_{p}}=1 / p$.

(ii) If $E=L_{p}(d t / t)$ then $\left\|D_{s} f\right\|=\left(\int_{0}^{\infty}|f(t / s)|^{p} d t / t\right)^{1 / p}=\|f\|$ and $\left\|D_{s} f\right\|=$ 1. So in this case we have $\alpha_{E}=\beta_{E}=0$.

(iii) If $E=L_{p}\left(t^{-\theta p} d t / t\right)$ for $\theta \in(0,1)$ then $\left\|D_{s} f\right\|=\left(\int_{0}^{\infty}|f(t / s)|^{p} t^{-\theta p} \frac{d t}{t}\right)^{1 / p}$ $=s^{-\theta}\|f\|$ and $\left\|D_{s} f\right\|=s^{-\theta}$. So in this case we have $\alpha_{E}=\beta_{E}=-\theta$.

Let us denote by

$L_{\infty}^{a}=\left\{f \in L_{0}\left(\mathbb{R}_{+}, d t / t\right): \operatorname{ess} \sup t^{-a}|f(t)|<\infty\right\}, \quad\|f\|_{L_{\infty}^{a}}=\operatorname{ess} \sup t^{-a}|f(t)|$. 
In the case $a=0$, we have $L_{\infty}=L_{\infty}^{0}$. These are Banach function spaces satisfying $\left\|D_{s}\right\|=s^{-a}$ but are not admissible because $\mathcal{C}_{00}$ fails to be dense. However, we can define $\hat{L}_{\infty}^{a}$ to be the closure of $\mathcal{C}_{00}$ in $L_{\infty}^{a}$ and this is admissible.

We recall that a pair of Banach spaces $\left(X_{0}, X_{1}\right)$ is called a Banach couple if $X_{0}, X_{1}$ are both continuously embedded in a Hausdorff topological vector space $V$. A Banach space $X$ also continuously embedded in $V$ is an intermediate space if $X_{0} \cap X_{1} \hookrightarrow$ $X \hookrightarrow X_{0}+X_{1}$. An intermediate space $X$ is called regular if $X_{0} \cap X_{1}$ is dense in $X$. An intermediate space $X$ is an interpolation space for the couple if for every linear map $T: X_{0}+X_{1} \longrightarrow X_{0}+X_{1}$ which restricts to a bounded operator on $X_{0}$ and $X_{1}$ is also a bounded operator on $X$. In this case, we get an estimate

$$
\|T\|_{X} \leq C \max \left\{\|T\|_{X_{0}},\|T\|_{X_{1}}\right\} .
$$

The K-functional for the couple $\left(X_{0}, X_{1}\right)$ is defined by

$$
K(t, x)=K\left(t, x ; X_{0}, X_{1}\right)=\inf \left\{\left\|x_{0}\right\|_{X_{0}}+t\left\|x_{1}\right\|_{X_{1}}: x=x_{0}+x_{1}\right\} .
$$

It is easy to check that for fixed $x$ the function $t \rightarrow K(t, x)$ is increasing and concave for and each $x \rightarrow K(t, x)$ gives an equivalent norm on the sum space. An intermediate space $X$ is called $K$-monotone or a real interpolation space if for some constant $C$ we have that if $y \in X$ and $x \in X_{0}+X_{1}$,

$$
K(t, x) \leq K(t, y), \quad 0<t<\infty, \Longrightarrow x \in X \quad \text { and } \quad\|x\|_{X} \leq C\|y\|_{X} .
$$

It is immediate that every K-monotone intermediate space is an interpolation space.

If $E$ is a Banach function space containing $\min (1, t)$ then we can define $X=$ $\left(X_{0}, X_{1}\right)_{E}$ to be the space of all $x \in X_{0}+X_{1}$ such that $K(t, x) \in E$ with the norm

$$
\|x\|_{X}=\|K(t, x)\|_{E, t} .
$$

This is clearly a K-monotone interpolation space. It is a fundamental result of Brudnyi and Krugljak [8] that every K-monotone interpolation space is (up to equivalence of norm) of this form and that $E$ can be chosen to be an interpolation space for the Banach couple $\left(L_{\infty}, L_{\infty}^{1}\right)$. This in turn means that by interpolation $-1 \leq \alpha_{E} \leq \beta_{E} \leq 0$. The key ingredient of this result is the following principle of K-divisibility fits proved by Brudnyi and Krugljak ([7,8]) and later refined in [10-12].

Theorem 2.2 There is an absolute constant $\gamma<6$ with the following property. Suppose $\left(X_{0}, X_{1}\right)$ is a Banach couple. Suppose $x \in X_{0}+X_{1}$ and $K(t, x) \leq \sum_{n=1}^{\infty} \psi_{n}(t)$ for all $t>0$, where each $\psi_{n}(t)$ is a positive concave function on $(0, \infty)$ and $\sum_{n=1}^{\infty} \psi_{n}(1)<\infty$. Then there exists a sequence of elements $\left\{x_{n}\right\} \subset X_{0}+X_{1}$ such that $x=\sum_{n=1}^{\infty} x_{n}$ in $X_{0}+X_{1}$ and

$$
K\left(t, x_{n}\right) \leq \gamma \psi_{n}(t)
$$

for $t>0$ and $n \in \mathbb{N}$. 
For the choice $E=L_{p}\left(t^{-\theta p} d t / t\right)$ where $0<\theta<1$ and $1 \leq p<\infty$ as in (iii) above we get the $(\theta, p)$-methods. If we take $E=L_{\infty}^{\theta}$ we get the $(\theta, \infty)$-method; if we take $E=\hat{L}_{\infty}^{\theta}$ we get the inner $(\theta, \infty)$-method. These methods were originally introduced by Lions and Peetre [20].

A $K$-monotone interpolation space $X$ is said to be of quasi-power type (respectively, admissible quasi-power type or a strict real interpolation space) if we can choose $E$ to satisfy the condition $-1<\alpha_{E} \leq \beta_{E}<0$ (respectively, to be admissible and satisfy $\left.-1<\alpha_{E} \leq \beta_{E}<0\right)$.

Proposition 2.3 If $\left(X_{0}, X_{1}\right)$ is a Banach couple then any strict real interpolation space is regular.

Proof Let $X=\left(X_{0}, X_{1}\right)_{E}$ is a strict real interpolation space where $E$ is admissible and $-1<\alpha_{E}<\beta_{E}<0$. Then for $x \in X$ we have that $\sum_{n=1}^{\infty} K\left(t / 2^{n}, x\right) \in E$ and $\sum_{n=1}^{\infty} 2^{-n} K\left(2^{n} t, x\right) \in E$. Note that by the monotonicity and concavity of the $\mathrm{K}$-functional these series must converge uniformly on compact subsets of $(0, \infty)$. For $n \in \mathbb{Z}$, let us write $x=u_{n}+v_{n}$ where $u_{n} \in X_{0}, v_{n} \in X_{1}$ and $\left\|u_{n}\right\|_{X_{0}}+2^{n}\left\|v_{n}\right\|_{X_{1}} \leq$ $2 K\left(2^{n}, x\right)$. Then $y_{n}=u_{n}-u_{n-1}=v_{n-1}-v_{n} \in X_{0} \cap X_{1}$ and

$$
\begin{aligned}
\left\|y_{n}\right\|_{X_{0}} \leq & 2 K\left(2^{n}, x\right),\left\|y_{n}\right\|_{X_{1}} \leq 4.2^{-n} K\left(2^{n-1}, x\right) \\
& +2.2^{-n} K\left(2^{n}, x\right) \leq 6.2^{-n} K\left(2^{n}, x\right)
\end{aligned}
$$

Thus

$$
K\left(t, y_{n}\right) \leq 6 \min \left(1,2^{-n} t\right) K\left(2^{n}, x\right), \quad n \in \mathbb{Z} .
$$

It follows that

$$
\sum_{n \in \mathbb{Z}} K\left(t, y_{n}\right) \leq 6 \sum_{n \in \mathbb{Z}} \min \left(1,2^{-n} t\right) K\left(2^{n}, x\right) \leq 12 \sum_{n \in \mathbb{Z}} \min \left(1,2^{-n}\right) K\left(2^{n} t, x\right) .
$$

In particular $\sum_{n \in \mathbb{Z}} K\left(t, y_{n}\right) \in E$.

The sequence $\sum_{|n| \geq N} K\left(t, y_{n}\right)$ converges uniformly to 0 on compact subsets of $(0, \infty)$ and hence by Lemma 2.1 we have

$$
\lim _{N \rightarrow \infty}\left\|\sum_{|n| \geq N} K\left(t, y_{n}\right)\right\|_{E}=0
$$

Thus the sequence $\left(\sum_{n=-N}^{N} y_{n}\right)_{N=1}^{\infty}$ converges in $X$. Note also that

$$
\lim _{n \rightarrow-\infty}\left\|u_{n}\right\|_{X_{0}} \leq 2 \lim _{n \rightarrow-\infty} K\left(2^{n}, x\right)=0
$$


and

$$
\lim _{n \rightarrow \infty}\left\|v_{n}\right\|_{X_{1}} \leq 2 \lim _{n \rightarrow \infty} 2^{-n} K\left(2^{n}, x\right)=0
$$

Hence $\sum_{n \in \mathbb{Z}} y_{n}=x$ in $X_{0}+X_{1}$ so that $x \in X$.

Theorem 2.4 Let $\left(X_{0}, X_{1}\right)$ be a Banach couple and let $X$ be an intermediate space. Then the following conditions on $X$ are equivalent:

(i) $X$ is a quasi-power type interpolation space for $\left(X_{0}, X_{1}\right)$.

(ii) There exists $0<\delta<1 / 2$ and a constant $C$ so that if $y \in X, x \in X_{0}+X_{1}$ and $s>0$,

$$
\begin{gathered}
K(t, x) \leq K(s t, y), \quad 0<t<\infty \Rightarrow x \in X \\
\text { and }\|x\|_{X} \leq C \max \left(s^{1-\delta}, s^{\delta}\right)\|y\|_{X} .
\end{gathered}
$$

If, further $X$ is regular then (ii) is equivalent to

(iii) $X$ is a strict real interpolation space for $\left(X_{0}, X_{1}\right)$.

Proof (i) $\Longrightarrow$ (ii). Suppose $X=\left(X_{0}, X_{1}\right)_{E}$ where $E$ is an admissible function space with $-1<\alpha_{E} \leq \beta_{E}<0$. Choose $-1<a<\alpha_{E}$ and $\beta_{E}<b<0$. Suppose $y \in X$ and $x \in X_{0}+X_{1}$ are such that $K(t, x) \leq K(s t, y)$ for some $s>0$ and all $0<t<\infty$. Since $K(t, x) \leq K(s t, y)$ for all $t>0$ and $K(s t, y) \in E$ we conclude that $K(t, x) \in E$ and hence

$$
\begin{aligned}
\|x\|_{X}=\|K(t, x)\|_{E} & \leq\|K(s t, y)\|_{E} \leq\left\|D_{1 / s}\right\|_{E}\|K(t, y)\|_{E} \\
& \leq C \max \left(s^{-a}, s^{-b}\right)\|y\|_{X} .
\end{aligned}
$$

Choosing $\delta=\min (-b, 1+a, 1 / 2)$ we obtain

$$
\|x\|_{X} \leq C \max \left(s^{1-\delta}, s^{\delta}\right)\|y\|_{X} .
$$

(ii) $\Longrightarrow$ (i). We define $E$ to be the space of $f \in L_{\infty}+L_{\infty}^{1}$ such that there exist $x_{j} \in X, s_{j}>0$ with $\sum_{j=1}^{\infty} \max \left(s_{j}^{1-\delta}, s_{j}^{\delta}\right)\left\|x_{j}\right\|<\infty$ and

$$
|f(t)| \leq \sum_{j=1}^{\infty} K\left(s_{j} t, x_{j}\right) \quad 0<t<\infty
$$

We define

$$
\|f\|_{E}=\inf \sum_{j=1}^{\infty} \max \left(s_{j}^{1-\delta}, s_{j}^{\delta}\right)\left\|x_{j}\right\|
$$

where the infimum is taken over all representations of the form of (2.6). 
Now if $x \in X$ it is clear that $K(t, x) \in E$ and $\|K(t, x)\|_{E} \leq\|x\|_{X}$. Conversely if $x \in X_{0}+X_{1}$ and $K(t, x) \in E$ then we can find $y_{j} \in X$ and $s_{j}>0$ with

$$
\sum_{j=1}^{\infty} \max \left(s_{j}^{1-\delta}, s_{j}^{\delta}\right)\left\|y_{j}\right\|<2\|K(t, x)\|_{E}
$$

and

$$
K(t, x) \leq \sum_{j=1}^{\infty} K\left(s_{j} t, y_{j}\right) \quad 0<t<\infty .
$$

By the Principle of K-divisibility (Theorem 2.2), for some absolute constant $\gamma$ we can write $x=\sum_{j=1}^{\infty} x_{j}$ in $X_{0}+X_{1}$ where

$$
K\left(t, x_{j}\right) \leq \gamma K\left(s_{j} t, y_{j}\right) \quad 0<t<\infty
$$

Thus $x_{j} \in X$ and $\left\|x_{j}\right\|_{X} \leq C \gamma \max \left(s_{j}^{1-\delta}, s_{j}^{\delta}\right)\left\|y_{j}\right\|_{X}$ and so $x \in X$ with $\|x\|_{X} \leq$ $2 C \gamma\|K(t, x)\|_{E, t}$.

Now suppose $f \in E$ and $s>0$. Then we can find $s_{j}>0$ and $x_{j} \in X$ so that

$$
|f(t)| \leq \sum_{j=1}^{\infty} K\left(s_{j} t, x_{j}\right) \quad 0<t<\infty
$$

and

$$
\sum_{j=1}^{\infty} \max \left(s^{1-\delta}, s^{\delta}\right)\left\|x_{j}\right\|_{X} \leq 2\|f\|_{E} .
$$

Now

$$
|f(t / s)| \leq \sum_{j=1}^{\infty} K\left(s_{j} s^{-1} t, x_{j}\right) \quad 0<t<\infty
$$

and so

$$
\begin{aligned}
\left\|D_{s} f\right\|_{E} & =\|f(t / s)\|_{E} \leq \sum_{j=1}^{\infty} \max \left(\left(s_{j} s^{-1}\right)^{1-\delta},\left(s_{j} s^{-1}\right)^{\delta}\right)\left\|x_{j}\right\| \\
& \leq \max \left(s^{\delta-1}, s^{-\delta}\right) \sum_{j=1}^{\infty} \max \left(s_{j}^{1-\delta}, s_{j}^{\delta}\right)\left\|x_{j}\right\| .
\end{aligned}
$$


This implies $\left\|D_{s}\right\|_{E} \leq \max \left(s^{\delta-1}, s^{-\delta}\right)$, and using $\delta<1 / 2$ we get

$$
\delta-1 \leq \alpha_{E} \leq \beta_{E} \leq-\delta
$$

(ii) $\Longrightarrow$ (iii) when $X$ is regular. We show that $E$ constructed in the previous proof is admissible. This requires only that $\mathcal{C}_{00}$ is dense in $E$ and for this we only need show that if $x \in X$ then $K(t, x)$ is in the closure of $\mathcal{C}_{00}$. First for $\epsilon>0$ pick $y \in X_{0} \cap X_{1}$ so that $\|x-y\|_{X}<\epsilon$. Then $\|K(t, x)-K(t, y)\|_{E}<\epsilon$. However, $K(t, y) \leq C \min (1, t)$ for some constant $C$. Now for $\tau>1$ we have

and

$$
\left\|K(t, y) \chi_{\left[0, \tau^{-1}\right)}\right\|_{E} \leq C\left\|\min (1, t) \chi_{\left(0, \tau^{-1}\right)}\right\|_{E} \leq C \tau^{-1}\left\|\chi_{(0,1)}\right\|_{E}
$$

$$
\left\|K(t, y) \chi_{(\tau, \infty)}\right\|_{E} \leq C\left\|D_{\tau}\right\|_{E}\left\|\chi_{(1, \infty)}\right\|_{E} \leq C \max \left(\tau^{\delta-1}, \tau^{-\delta}\right)\left\|\chi_{(1, \infty)}\right\|_{E} .
$$

It follows that $\lim _{\tau \rightarrow \infty}\left\|K(t, y)-K(t, y) \chi_{\left[\tau^{-1}, \tau\right]}\right\|_{E}=0$ and hence that $\lim \sup _{\tau \rightarrow \infty}\left\|K(t, x)-K(t, x) \chi_{\left[\tau^{-1}, \tau\right]}\right\|_{E}<\epsilon$.

\section{Sectorial operators}

We employ standard notation from Banach space theory. Throughout, $X$ denotes a complex Banach space.

A sector of angle $0<\phi<\pi$ in the complex plane is the open set defined by

$$
\Sigma_{\phi}=\{\lambda \in \mathbb{C} \backslash\{0\}:|\arg \lambda|<\phi\} .
$$

A closed operator $A$ on $X$ is called sectorial if:

(i) $A$ is one-to-one.

(ii) The domain $\operatorname{Dom}(A)$ and the range $\operatorname{Ran}(A)$ are dense in $X$.

(iii) There exists $0<\phi<\pi$ so that the spectrum $\operatorname{Sp}(A)$ is contained in $\overline{\Sigma_{\phi}}$ and one has the resolvent estimate:

$$
\|\lambda R(\lambda, A)\| \leq C, \quad \lambda \in \mathbb{C} \backslash \overline{\Sigma_{\phi}} .
$$

Notice that this definition does not require $A$ to be invertible. It follows from this definition that $A^{-1}$ is also a sectorial operator. We define the angle of sectoriality of $A$ by letting $\omega(A)$ be the infimum of all $\phi$ so that (3.8) holds.

We denote by $H^{\infty}\left(\Sigma_{\phi}\right)$ the space of all bounded analytic functions on the sector $\Sigma_{\phi}$ where $0<\phi<\pi$. We define $H_{0}^{\infty}\left(\Sigma_{\phi}\right)$ to be the space of all $f \in H^{\infty}\left(\Sigma_{\phi}\right)$ which obey the estimate of the form $|f(z)| \leq C \frac{|z|^{\delta}}{(1+|z|)^{2 \delta}}$ with $\delta>0$.

If $a, b>0$ we defined

$$
\varphi_{a, b}(z)=\frac{z^{a}}{(1+z)^{a+b}} .
$$

This function is in $H_{0}^{\infty}\left(\Sigma_{\phi}\right)$ for every choice of $0<\phi<\pi$. 
For any $\phi>\omega(A)$ suppose $f \in H_{0}^{\infty}\left(\Sigma_{\phi}\right)$. Then, we can define $f(A)$ as a bounded operator by a contour integral i.e.,

$$
f(A)=\frac{1}{2 \pi i} \int_{\Gamma_{v}} f(\zeta) R(\zeta, A) d \zeta
$$

where $\phi>v>\omega(A)$ and $\Gamma_{v}=\left\{|t| e^{-i(\operatorname{sgn} t) v}:-\infty<t<\infty\right\}$. Notice that we get an estimate:

$$
\|f(t A)\| \leq C \int_{-\infty}^{\infty}\left|f\left(s e^{i(\operatorname{sgn} s) v}\right)\right| \frac{d s}{|s|}
$$

where $C=C(v, A)$. The map $f \rightarrow f(A)$ is an algebra homomorphism from $H_{0}^{\infty}\left(\Sigma_{\phi}\right)$ into $\mathcal{L}(X)$.

The following Lemma will be used many times in the sequel:

Lemma 3.1 Suppose $a, b>0$ and $f, g \in H^{\infty}\left(\Sigma_{\phi}\right)$ where $\phi>\omega(A)$. Suppose that $f, g$ satisfy $|f(z)| \leq|z|^{a}(1+|z|)^{-a-b}$ and $|g(z)| \leq|z|^{a^{\prime}}(1+|z|)^{-a^{\prime}-b^{\prime}}$ where $a, a^{\prime}, b, b^{\prime}>0$. Then there exists a constant $C=C\left(A, a, b, a^{\prime}, b^{\prime}\right)$ so that

$$
\|f(u t A) g(t A)\| \leq \begin{cases}C u^{\min \left(a, b^{\prime}\right)} & t>0, \quad 0<u \leq 1, \quad a \neq b^{\prime} \\ C u^{a}(1+|\log u|) & t>0, \quad 0<u \leq 1, \quad a=b^{\prime} \\ C u^{-\min \left(a^{\prime}, b\right)} & t>0, \quad 1 \leq u<\infty, \quad a^{\prime} \neq b \\ C u^{-b}(1+|\log u|) & t>0, \quad 1 \leq u<\infty, \quad a^{\prime}=b .\end{cases}
$$

Proof By (3.10) for some $\phi>v>\omega(A)$ we have

$$
\begin{aligned}
\|f(u t A) g(t A)\| & \leq C \int_{-\infty}^{\infty}\left|f\left(u s e^{-i v \operatorname{sgn}(s)}\right) g\left(s e^{-i v \operatorname{sgn}(s)}\right)\right| \frac{d s}{|s|} \\
& \leq C u^{a} \int_{0}^{\infty} s^{a+a^{\prime}}(1+u s)^{-(a+b)}(1+s)^{-\left(a^{\prime}+b^{\prime}\right)} \frac{d s}{s}
\end{aligned}
$$

Suppose $u \geq 1$. Then since $(1+u s)^{-(a+b)}(1+s)^{-\left(a^{\prime}+b^{\prime}\right)}<1$ we have,

$$
\int_{0}^{u^{-1}} s^{a+a^{\prime}-1}(1+u s)^{-(a+b)}(1+s)^{-\left(a^{\prime}+b^{\prime}\right)} d s \leq \int_{0}^{u^{-1}} s^{a+a^{\prime}-1} d s=\frac{1}{a+a^{\prime}} u^{-a-a^{\prime}}
$$


Since $(1+u s)^{-(a+b)} \leq(u s)^{-(a+b)}$ we get

$$
\begin{aligned}
\int_{u^{-1}}^{1} s^{a+a^{\prime}-1}(1+u s)^{-(a+b)}(1+s)^{-\left(a^{\prime}+b^{\prime}\right)} d s & \leq u^{-(a+b)} \int_{u^{-1}}^{1} s^{a^{\prime}-b-1} d s \\
& =\left\{\begin{array}{l}
\frac{1}{a^{\prime}-b}\left(u^{-a-b}-u^{-a-a^{\prime}}\right) \text { if } a^{\prime} \neq b \\
u^{-a-b} \log u \text { if } a^{\prime}=b
\end{array}\right.
\end{aligned}
$$

For the last integral we also use that $(1+s)^{-\left(a^{\prime}+b^{\prime}\right)}<s^{-\left(a^{\prime}+b^{\prime}\right)}$

$$
\int_{1}^{\infty} s^{a+a^{\prime}-1}(1+u s)^{-(a+b)}(1+s)^{-\left(a^{\prime}+b^{\prime}\right)} d s \leq u^{-(a+b)} \int_{1}^{\infty} s^{-1-b-b^{\prime}} d s=\frac{1}{b^{\prime}+b} u^{-a-b}
$$

Since $u \geq 1$, we have $u^{-a^{\prime}} \leq u^{-\min \left(a^{\prime}, b\right)}$ and $u^{-b} \leq u^{-\min \left(a^{\prime}, b\right)}$. Therefore for a suitable constant $C>0$, we obtain the last two estimates. If $u \leq 1$ we observe that $f(u t A) g(t A)=f(s A) g\left(u^{-1} s A\right)$ where $s=u t$. Combining these two estimates we obtain the Lemma.

If $f \in H^{\infty}\left(\Sigma_{\phi}\right)$ then (3.9) does not necessarily converge as a Bochner integral. However for every $g \in H_{0}^{\infty}\left(\Sigma_{\phi}\right)$ we can define the operator $(f g)(A)$. Now if $x \in \operatorname{Dom}(A) \cap \operatorname{Ran}(A)$ then $x=\varphi_{1,1}(A) y$ for some $y \in X$. Therefore, we can define $f(A) x=\left(f \varphi_{1,1}\right)(A) y$.

If we assign

$$
v_{n}(z)=\frac{n}{n+z}-\frac{1}{1+n z}
$$

then $v_{n}(A)=\left(n-\frac{1}{n}\right) A R(-n, A) R\left(-\frac{1}{n}, A\right)$ maps $X$ into $\operatorname{Dom}(A) \cap \operatorname{Ran}(A)$ and it may be shown that $\left(f v_{n}\right)(A) x=f(A) v_{n}(A) x$. If $\sup _{n}\left\|\left(v_{n} f\right)(A)\right\|<\infty$ then we can define

$$
f(A) x=\lim _{n \rightarrow \infty}\left(v_{n} f\right)(A) x \quad x \in X
$$

as a bounded operator. This is equivalent to the fact that $f(A)$ satisfies an estimate

$$
\|f(A) x\| \leq C\|x\| \quad x \in \operatorname{Dom}(A) \cap \operatorname{Ran}(A) .
$$

As an alternative way to view this procedure, one can densely define $f(A)$ by

$$
f(A) x=\frac{1}{2 \pi i} \int_{\Gamma_{v}} f(\zeta) \zeta^{-\frac{1}{2}} A^{\frac{1}{2}} R(\zeta, A) x d \zeta, \quad x \in \operatorname{Dom}(A) \cap \operatorname{Ran}(A)
$$


where $A^{\frac{1}{2}} R(\zeta, A)$ is a well-defined operator since $z^{\frac{1}{2}}(\zeta-z)^{-1}$ belongs to $H_{0}^{\infty}\left(\Sigma_{\phi^{\prime}}\right)$ where $\omega<\phi^{\prime}<v$. Then $f(A)$ extends to a bounded operator if one has a norm estimate

$$
\|f(A) x\| \leq C\|x\| \quad x \in \operatorname{Dom}(A) \cap \operatorname{Ran}(A) .
$$

If $f(A)$ is bounded for all $f \in H^{\infty}\left(\Sigma_{\phi}\right)$, we say that $A$ has $H^{\infty}\left(\Sigma_{\phi}\right)$-calculus. We then have an estimate

$$
\|f(A)\| \leq C\|f\|_{H^{\infty}\left(\Sigma_{\phi}\right)} \quad f \in H^{\infty}\left(\Sigma_{\phi}\right)
$$

We can define the corresponding angle of $H^{\infty}$-calculus by letting $\omega_{H}(A)$ be the infimum of all $\phi$ so that $A$ has an $H^{\infty}\left(\Sigma_{\phi}\right)$-calculus. See $[9,18]$ for details.

We now describe an abstract framework for defining operators $f(A)$ for more general analytic functions. Let us define the bounded operator $T=\varphi_{1,1}(A)$. Then it is possible to define a linear space $\mathcal{X}$ containing $X$ so that $T$ extends to a linear bijection on $\mathcal{X}$. Indeed let $\mathcal{Y}$ be the space of sequences $\left(x_{n}\right)_{n=0}^{\infty}$ where $x_{n} \in X$ so that $T x_{n}=x_{n+1}$ eventually. Let $\mathcal{X}$ be the space obtained by factoring out the subspace of all $\left(x_{n}\right)$ so that $x_{n}=0$ eventually. Then $X$ can be identified with the subspace generated by the sequences $\left(T^{n} x\right)_{n=0}^{\infty}$ where $x \in X$ and $T$ extends to the operator induced by the shift $\left(x_{n}\right)_{n=0}^{\infty} \rightarrow\left(x_{n+1}\right)_{n=0}^{\infty}$. Notice that $\mathcal{X}=\cup_{m=0}^{\infty} T^{-m} X$. In concrete situations where $X$ is already embedded in some topological vector space $V$ (e.g., a space of distributions) on which $T$ is an isomorphism we can simply identify $\mathcal{X}$ with $\cup_{m=0}^{\infty} T^{-m} X \subset V$.

Now suppose $f$ is an analytic function on $\Sigma_{\phi}$ where $\phi>\omega(A)$ which satisfies

$$
|f(z)| \leq C \max \left(|z|^{m},|z|^{-m}\right) \quad z \in \Sigma_{\phi}
$$

where $m \in \mathbb{N}$. Then $f(A) T^{n}$ is a well-defined bounded operator if $n \geq m+1$ and we can induce an operator $f(A)$ on $\mathcal{X}$ by putting

$$
f(A)\left(x_{n}\right)_{n=0}^{\infty}=\left(y_{n}\right)_{n=0}^{\infty}
$$

where $y_{n}=f(A) x_{n}$ if $x_{n} \in T^{m+1}(X)$ and 0 otherwise. Then if $\operatorname{Dom} f(A)=\{x \in$ $X: f(A) x \in X\} f(A)$ defines a closed operator on $X$ which becomes a bounded operator if $\operatorname{Dom} f(A)=X$. It is easy to show that the map $f \rightarrow f(A)$ is an algebraic homomorphism into the algebra $\mathcal{L}(\mathcal{X})$ of all linear maps on $\mathcal{X}$.

If $-\infty<\sigma<\infty$, we thus may define the fractional powers $A^{\sigma} . A^{\sigma}$ is one-one with dense domain and range on $X$; it is sectorial if and only if $|\sigma| \omega(A)<\pi$ and then $\omega\left(A^{\sigma}\right)=|\sigma| \omega(A)$. We define $\mathcal{D}\left(A^{\sigma}\right)$ as the space $A^{-\sigma}(X) \subset \mathcal{X}$ under the norm $x \rightarrow\left\|A^{\sigma} x\right\|$. Then $\operatorname{Dom}\left(A^{\sigma}\right)$ is dense in $\mathcal{D}\left(A^{\sigma}\right)$.

We now generalize these ideas to operator-valued analytic functions, following ideas in [18]. We denote by $\mathcal{A}$ the algebra of all bounded operators which commute with $R(\lambda, A)$ for all $\lambda$ in the resolvent set. For $\omega(A)<\phi<\pi$, we define $H^{\infty}\left(\Sigma_{\phi}, \mathcal{A}\right)$ as the space of all bounded analytic functions $F: \Sigma_{\phi} \longrightarrow \mathcal{A}$, so that for every $x \in X$ the map $z \rightarrow F(z) x$ is analytic (i.e., $F$ is analytic for the strong operator topology). 
We consider the scalar space $H^{\infty}\left(\Sigma_{\phi}\right)$ embedded as a subspace of $H^{\infty}\left(\Sigma_{\phi}, \mathcal{A}\right)$ via the identification $f \rightarrow f I$. We denote by $H_{0}^{\infty}\left(\Sigma_{\phi}, \mathcal{A}\right)$ the space of all $F \in H^{\infty}\left(\Sigma_{\phi}, \mathcal{A}\right)$ which obey an estimate of the form $\|F(z)\| \leq C \varphi_{\delta, \delta}(|z|)$ for some $\delta>0$.

We can then define $F(A)$ for $F \in H_{0}^{\infty}\left(\Sigma_{\phi}\right)$ by the same formula:

$$
f(A)=\frac{1}{2 \pi i} \int_{\Gamma_{v}} F(\zeta) R(\zeta, A) d \zeta
$$

where $\Gamma_{v}=\left\{|t| e^{-i(\operatorname{sgn} t) v}:-\infty<t<\infty\right\}$. If $F \in H^{\infty}\left(\Sigma_{\phi}\right)$ we similarly define $F(A)$ as a closed operator with dense range which is bounded if we have an estimate

$$
\|F(A) x\| \leq C\|x\| \quad x \in \operatorname{Dom}(A) \cap \operatorname{Ran}(A) .
$$

\section{Operators with an absolute functional calculus}

Let $A$ be a sectorial operator on $X$. We say that $A$ has an absolute functional calculus if there are functions $g, h \in H_{0}^{\infty}\left(\Sigma_{\phi}\right)$ where $\phi>\omega(A)$ so that for some constant $C$ we have the following implication

$$
\|h(t A) g(t A) x\| \leq\|g(t A) y\| \quad 0<t<\infty \Rightarrow\|x\| \leq C\|y\| .
$$

It we pick $\delta>0$ so that $h(z) / \varphi_{\delta, \delta}(z) \in H_{0}^{\infty}\left(\Sigma_{\phi}\right)$ it is clear that we may replace $h$ by $\varphi_{\delta, \delta}$ in this definition. We say that $A$ has an $(a, b)$-absolute functional calculus if (4.13) holds for the the choice $g(z)=\varphi_{a, b}(z)$. Thus $A$ has an $(a, b)$-absolute functional calculus if for some $\delta>0, C>0$ we have

$$
\left\|\varphi_{a+\delta, b+\delta}(t A) x\right\| \leq\left\|\varphi_{a, b}(t A) y\right\| \quad 0<t<\infty \Rightarrow\|x\| \leq C\|y\| .
$$

Let us first show the following elementary fact:

Proposition 4.1 If $A$ is an invertible sectorial operator satisfying (4.14) then for any $t_{0}>0$ there exists $C_{1}=C_{1}\left(t_{0}\right)$ so that

$$
\left\|\varphi_{a+\delta, b+\delta}(t A) x\right\| \leq\left\|\varphi_{a, b}(t A) y\right\| \quad 0<t \leq t_{0} \Rightarrow\|x\| \leq C_{1}\|y\|
$$

Proof There is a constant $C_{2}$ so that for $t \geq t_{0}$ we have $\left\|S_{t}\right\|,\left\|S_{t}^{-1}\right\|,\left\|T_{t}\right\|,\left\|T_{t}^{-1}\right\| \leq$ $C_{2}$ where

$$
S_{t}=t^{(a+b)} A^{(a+b)}(1+t A)^{-(a+b)}, \quad T_{t}=t^{a+b+2 \delta} A^{a+b+2 \delta}(1+t A)^{-(a+b+2 \delta)} .
$$

Thus we have

$$
\left\|t_{0}^{-b-\delta} T_{t_{0}} A^{-b-\delta} x\right\| \leq\left\|t_{0}^{-b} S_{t_{0}} A^{-b} y\right\|
$$


which implies that

$$
\left\|A^{-b-\delta} x\right\| \leq C_{2}^{2} t_{0}^{\delta}\left\|A^{-b} y\right\|
$$

Now if $t \geq t_{0}$ we have

$$
\begin{aligned}
\left\|\varphi_{a+\delta, b+\delta}(t A) x\right\| & =\left\|T_{t}\left(t^{-(b+\delta)} A^{-(b+\delta}\right) x\right\| \\
& \leq C_{2} t^{-b-\delta}\left\|A^{-b-\delta} x\right\| \\
& \leq C_{2}^{3} t_{0}^{\delta} t^{-b-\delta}\left\|A^{-b} y\right\| \\
& =C_{2}^{3}\left(t_{0} / t\right)^{\delta}\left\|S_{t}^{-1} \varphi_{a, b}(t A) y\right\| \\
& \leq C_{2}^{4}\left\|\varphi_{a, b}(t A) y\right\|,
\end{aligned}
$$

and the Lemma follows with $C_{1}=C C_{2}^{4}$.

First, we establish that if $A$ has an absolute calculus then it has an $H^{\infty}$-calculus.

Theorem 4.2 Suppose that $A$ is a sectorial operator on $X$ with an absolute functional calculus. Then A has an $H^{\infty}$-calculus and $\omega_{H}(A)=\omega(A)$.

Proof Fix any $\phi>\omega(A)$ so that $g \in H_{0}^{\infty}\left(\Sigma_{\phi}\right)$ (where $g, h$ are the functions in the definition of the absolute calculus). Suppose $f \in H^{\infty}\left(\Sigma_{\phi}\right)$. Notice that for an appropriate contour $\Gamma_{v}$ with $\omega<v<\phi$ and $x \in \operatorname{Dom}(A) \cap \operatorname{Ran}(A)$,

$$
\begin{aligned}
\|h(t A) f(A) x\| & \leq(2 \pi)^{-1} \int_{\Gamma_{v}}\|h(t \zeta) f(\zeta) R(\zeta, A) x\| d|\zeta| \\
& \leq C\|f\|_{H^{\infty}\left(\Sigma_{\phi}\right)} \int_{\Gamma}|h(t \zeta)(\zeta)| \frac{d|\zeta|}{|\zeta|}\|x\| \\
& \leq C\|f\|_{H^{\infty}\left(\Sigma_{\phi}\right)} \int_{\Gamma}|h(t \zeta)| \frac{d|\zeta|}{|\zeta|}\|x\| \\
& \leq C\|f\|_{\infty}\|x\|
\end{aligned}
$$

Now it follows that

$$
\|h(t A) g(t A) f(A) x\| \leq C\|f\|_{H^{\infty}\left(\Sigma_{\phi}\right)}\|g(t A) x\| \quad x \in \operatorname{Dom}(A) \cap \operatorname{Ran}(A) .
$$

Thus, we have an estimate

$$
\|f(A) x\| \leq C\|f\|_{H^{\infty}\left(\Sigma_{\phi}\right)}\|x\| \quad x \in \operatorname{Dom}(A) \cap \operatorname{Ran}(A)
$$

and $A$ has an $H^{\infty}\left(\Sigma_{\phi}\right)$-calculus.

We now turn to examples. First let $X$ be a space with an unconditional basis $\left(e_{n}\right)_{n \in \mathbb{Z}}$. Define the sectorial operator $A$ by

$$
A x=\sum_{n \in \mathbb{Z}} 2^{n} e_{n}^{*}(x) e_{n}
$$


with the natural domain $\left\{x: \sum_{n \in \mathbb{Z}} 2^{n} e_{n}^{*}(x) e_{n}\right.$ converges $\}$. Then $A$ has an $H^{\infty}$-calculus and $\omega_{H}(A)=\{0\}$.

Proposition 4.3 A has an absolute functional calculus.

Proof Since $\left\{2^{n}\right\}$ is an interpolating sequence in any sector $\Sigma_{\phi}$, we can find $g \in$ $H_{0}^{\infty}\left(\Sigma_{\phi}\right)$ such that $g(1)=1$ and $g\left(2^{n}\right)=0$ for $n \in \mathbb{Z} \backslash\{0\}$. Suppose $x, y \in X$ and

$$
\left\|g(t A)^{2} x\right\| \leq\|g(t A) y\| \quad 0<t<\infty .
$$

Then taking $t=2^{-n}$ for $n \in \mathbb{Z}$ we have

$$
\left|e_{n}^{*}(x)\right| \leq\left|e_{n}^{*}(y)\right| \quad n \in \mathbb{Z}
$$

and so

$$
\|x\| \leq C\|y\|
$$

where $C$ is the unconditional basis constant.

Example Suppose $X$ is the space of sequences with the norm

$$
\left\|\sum_{j \in \mathbb{Z}} \xi_{j} e_{j}\right\|=\left(\sum_{j \in \mathbb{Z}}\left|\xi_{2 j}\right|^{2}\right)^{1 / 2}+\left(\sum_{j \in \mathbb{Z}}\left|\xi_{2 j-1}\right|^{p}\right)^{1 / p}
$$

where $\left\{e_{n}\right\}$ is a canonical basis and $1<p<2$. Then $A$ cannot have an $(a, b)$-absolute functional calculus for any $a, b>0$. In fact if we let $y=e_{2}+e_{4}+\cdots+e_{2 n}$ and $x=e_{1}+e_{3}+\cdots+e_{2 n-1}$ it is easy to verify that

$$
\left\|\varphi_{a, b}(t A) x\right\| \leq C\left\|\varphi_{a, b}(t A) y\right\| \quad 0<t<\infty
$$

where $C=C(a, b)$ is an absolute constant independent of $n$. This implies that for any given $\delta>0$ we have

$$
\left\|\varphi_{a+\delta, b+\delta}(t A) x\right\| \leq C_{\delta}\left\|\varphi_{a, b}(t A) y\right\| \quad 0<t<\infty
$$

since $\left\{\varphi_{\delta, \delta}(t A)\right\}_{t>0}$ is a uniformly bounded family of operators. However $\|y\|=n^{1 / 2}$ and $\|x\|=n^{1 / p}$.

Part of the motivation for the study of operators with an absolute functional calculus is the fact that for certain Banach spaces a sectorial operator has an $H^{\infty}$-calculus if and only if it has an absolute functional calculus.

Theorem 4.4 Let X be a Hilbert space and let A be a sectorial operator on X. If A has an $H^{\infty}$-calculus then $A$ has an $(a, b)$-absolute functional calculus for any $a, b>0$. 
Proof This is essentially due to McIntosh [22] (or see [3]). It is an immediate consequence of the fact that if $a, b>0$ we have an equivalence:

$$
\|x\| \approx\left(\int_{0}^{\infty}\left\|\varphi_{a, b}(t A) x\right\|^{2} \frac{d t}{t}\right)^{1 / 2} .
$$

Corresponding results hold for sectorial operators on $L_{1}$ - and $\mathcal{C}(K)$-spaces, and these are essentially contained in [18]. Recall that a Banach space $X$ is a GT-space if every bounded operator $T: X \longrightarrow \ell_{2}$ is absolutely summing, i.e., there exists $K>0$ such that

$$
\sum_{k=1}^{n}\left\|T x_{k}\right\| \leq K \max _{\alpha_{k}= \pm 1}\left\|\sum_{k=1}^{n} \alpha_{k} x_{k}\right\|
$$

for every collection $x_{1}, \ldots, x_{n} \in X$. Important examples of GT-spaces are $L_{1}$ and $L_{1} / H_{1}$ (see [5,6,23]). Examples of spaces for which the dual is a GT-space are $C(K)$ and the disc algebra $A(\mathbb{D})$.

Theorem 4.5 Let $X$ be a Banach space so that either (i) X is a GT-space, or (ii) $X^{*}$ is a GT-space. Suppose A is a sectorial operator on $X$ with an $H^{\infty}$-calculus; then $A$ has an $(a, b)$-absolute functional calculus for every $a, b>0$.

Proof (i) The proof of Proposition 7.1 of [18] gives that

$$
\|x\| \approx \int_{0}^{\infty}\left\|\varphi_{a, b}(t A) x\right\| \frac{d t}{t} .
$$

Note here that it is not necessary to assume $X$ has cotype two, since if $X$ is a GT-space then $X$ has the Orlicz property [23, Chapter 6c] and this is sufficient for (7.2) in [18]. (It is unknown whether every GT-space has cotype two.) The Theorem now follows as before.

(ii) A similar argument in this case shows that for the dual norm whenever $a, b>0$,

$$
\left\|x^{*}\right\| \approx \int_{0}^{\infty}\left\|\varphi_{a, b}(t A)^{*} x^{*}\right\| \frac{d t}{t} .
$$

Then for a suitable constant $C$ we have

$$
\int_{0}^{\infty}\left\|\varphi_{a, b}(t A)^{*} x^{*}\right\| \frac{d t}{t} \leq C\left\|x^{*}\right\|, \quad x^{*} \in X^{*} .
$$


Suppose $x \in X$.

$$
x=c \int_{0}^{\infty} \varphi_{2 a, 2 b}(t A) x \frac{d t}{t} \quad x \in X
$$

where $c^{-1}=\int_{0}^{\infty} \varphi_{2 a, 2 b}(t) \frac{d t}{t}$. Thus if $\left\|x^{*}\right\|=1$ is chosen so that $x^{*}(x)=\|x\|$ we have

$$
\begin{aligned}
\|x\| & =c \int_{0}^{\infty}\left\langle\varphi_{a, b}(t A) x, \varphi_{a, b}(t A)^{*} x^{*}\right\rangle \frac{d t}{t} \\
& \leq c\left(\max _{t>0}\left\|\varphi_{a, b}(t A) x\right\|\right)\left(\int_{0}^{\infty}\left\|\varphi_{a, b}(t A)^{*} x^{*}\right\| \frac{d t}{t}\right) \\
& \leq C c \max _{t>0}\left\|\varphi_{a, b}(t A) x\right\| .
\end{aligned}
$$

Thus

$$
\|x\| \approx \max _{t>0}\left\|\varphi_{a, b}(t A) x\right\|
$$

and the Theorem follows in this case.

\section{Spaces obtained by real interpolation}

Suppose $A$ is a sectorial operator on $X$ and $E$ is an admissible Banach function space. Let $0 \neq f \in H_{0}^{\infty}\left(\Sigma_{\phi}\right)$ where $\phi>\omega(A)$. We let $X_{E}(f)$ be the set of $x \in \mathcal{X}$ such that $f(t A) x \in X$ for every $t>0$ and $t \rightarrow\|f(t A) x\|_{X} \in E$ and we norm this space by

$$
\|x\|_{X_{E}(f)}=\|\| f(t A) x\|\|_{E, t} \quad x \in X .
$$

Theorem 5.1 Suppose $f$ and $g$ both satisfy estimates

$$
|f(z)|,|g(z)| \leq C|z|^{a}(1+|z|)^{-a-b} \quad z \in \Sigma_{\phi}
$$

where $a>\max \left(-\alpha_{E}, 0\right)$ and $b>\max \left(\beta_{E}, 0\right)$. Then $X_{E}(f)=X_{E}(g)$ and the norms $\|\cdot\|_{X_{E}(f)}$ and $\|\cdot\|_{X_{E}(g)}$ are equivalent.

Proof Let us define $f^{*}(z)=\overline{f(\bar{z})}$ for $z \in \Sigma_{\phi}$. Pick any $N>\max (a, b)$. Then

$$
\int_{0}^{\infty} f(t z) f^{*}(t z) \varphi_{N, N}(t z) \frac{d t}{t}=\int_{0}^{\infty}|f(t)|^{2} \varphi_{N, N}(t) \frac{d t}{t} \quad z \in \Sigma_{\phi}
$$


and by scaling we can assume this constant is one. Now by Lemma 3.1 we have

$$
\left\|\varphi_{N, N}(s t A) g(t A)\right\| \leq C \min \left(s^{b}, s^{-a}\right) \quad s, t>0 .
$$

Hence $g(t A) f(s t A) f^{*}(s t A) \varphi_{N, N}(s t A)$ is Bochner-integrable on $(0, \infty)$ with respect to $d s / s$ and clearly

$$
g(t A)=\int_{0}^{\infty} g(t A) f(s t A) f^{*}(s t A) \varphi_{N, N}(s t A) \frac{d s}{s} .
$$

For $x \in X_{E}(f)$ we have

$$
\|g(t A) x\| \leq C \int_{0}^{\infty} \min \left(s^{b}, s^{-a}\right)\|f(s t A) x\| \frac{d s}{s} .
$$

Thus if $G(t)=\|g(t A) x\|$ and $F(t)=\|f(t A) x\|$ we have

$$
G(t) \leq C \int_{0}^{\infty} \min \left(s^{b}, s^{-a}\right) D_{s^{-1}} F(t) \frac{d s}{s} .
$$

The map $s \rightarrow D_{s^{-1}} F$ is continuous from $(0, \infty)$ into $E$ by Lemma 2.1. Now

$$
\left\|D_{s^{-1}} F\right\|_{E} \leq C \max \left(s^{a^{\prime}}, s^{-b^{\prime}}\right)\|F\|_{E}
$$

where $a>a^{\prime}>-\alpha_{E}$ and $b>b^{\prime}>\beta_{E}$. Thus

$$
\|G\|_{E} \leq C\|F\|_{E} \int_{0}^{\infty} \min \left(s^{b-b^{\prime}}, s^{a^{\prime}-a}\right) \frac{d s}{s}
$$

which implies $x \in X_{E}(g)$ and an estimate

$$
\|x\|_{X_{E}(g)} \leq C\|x\|_{X_{E}(f)} .
$$

The converse estimate follows trivially by interchanging the roles of $f$ and $g$.

We can now define $X_{E}$ unambiguously as the space $X_{E}(f)$ as long as $f$ satisfies an estimate $|f(z)| \leq C|z|^{a}(1+|z|)^{-a-b}$ where $a>\max \left(0,-\alpha_{E}\right)$ and $b>\max \left(0, \beta_{E}\right)$.

Suppose $0>a>-\alpha_{E}$ or $0>b>\beta_{E}$ (only one can be true since $\alpha_{E} \leq \beta_{E}$ ). Fix $\gamma \in \mathbb{R}$ so that $\alpha_{E}+\gamma<0<\beta_{E}+\gamma$. We can define a new admissible Banach function space by $E_{\gamma}=\left\{f: t^{\gamma} f \in E\right\}$ with the norm $\|f\|_{E_{\gamma}}=\left\|t^{\gamma} f\right\|_{E}$. Then $\left\|D_{s}\right\|_{E_{\gamma}}=s^{\gamma}\left\|D_{s}\right\|_{E}$ and so $\alpha_{E_{\gamma}}=\alpha_{E}+\gamma$ and $\beta_{E_{\gamma}}=\beta_{E}+\gamma$. 
For a suitable choice of $f \in H_{0}^{\infty}\left(\Sigma_{\phi}\right),|f(z)| \leq C|z|^{a}(1+|z|)^{-a-b}$ consider $g(z)=z^{-\gamma} f(z)$. Then

$$
|g(z)| \leq C \frac{|z|^{a-\gamma}}{(1+|z|)^{(a-\gamma)+(b+\gamma)}}
$$

and we can define $X_{E_{\gamma}}(g)$ independent of $a, b$ and $g$ as long as $a>-\alpha_{E}, b>\beta_{E}$. We also have

$$
t^{\gamma}\|f(t A) x\|=\left\|g(t A) A^{\gamma} x\right\| \quad 0<t<\infty
$$

where it follows that $X_{E_{\gamma}}=A^{\gamma} X_{E}$.

Therefore if $0<\alpha_{E}$ or $\beta_{E}<0$ choose $\gamma$ so that $\alpha_{E}+\gamma \leq 0 \leq \beta_{E}+\gamma$ and then work with $A^{\gamma} X_{E}$. It is now possible to rewrite Theorem 5.1 in the form:

Theorem 5.2 Suppose $E$ is an admissible Banach function space and $f$ is an analytic function on $\Sigma_{\phi}$ where $\phi>\omega(A)$. Suppose $f$ satisfies the estimates

$$
|f(z)| \leq C|z|^{a}(1+|z|)^{-a-b} \quad z \in \Sigma_{\phi}
$$

where $a>-\alpha_{E}$ and $b>\beta_{E}$. Then

$$
X_{E}=\left\{x: f(t A) x \in X, 0<t<\infty \text { and }\|f(t A) x\|_{X} \in E\right\}
$$

and $\|x\|_{X_{E}} \approx\|\| f(t A) x\left\|_{X}\right\|_{E}$.

Note here that we no longer require $a, b>0$ and $f(t A)$ is regarded as an operator on $\mathcal{X}$. Of course it is natural to take $f(z)=\varphi_{a, b}$ as long as $a>-\alpha_{E}$ and $b>\beta_{E}$.

It follows that if $\sigma<\alpha_{E} \leq \beta_{E}<\tau$ then $X_{E}(f)$ can be identified with a subspace of $\mathcal{D}\left(A^{\sigma}\right)+\mathcal{D}\left(A^{\tau}\right)$. If $-\infty<\sigma<\tau<\infty$ then the pair $\left(\mathcal{D}\left(A^{\sigma}\right), \mathcal{D}\left(A^{\tau}\right)\right)$ is a Banach couple (for the ambient space $V$ we may take $T^{-m}(X)$ for large enough $m$ under the norm $\left.x \rightarrow\left\|T^{m} x\right\|\right)$.

Proposition 5.3 Suppose $a+b>0$. Then

$$
K\left(t, x ; \mathcal{D}\left(A^{-b}\right), \mathcal{D}\left(A^{a}\right)\right) \approx t^{\frac{b}{a+b}}\left\|\varphi_{a, b}\left(t^{\frac{1}{a+b}} A\right) x\right\|_{X} \quad x \in \mathcal{D}\left(A^{-b}\right)+\mathcal{D}\left(A^{a}\right) .
$$

Proof We will show that $\left(\mathcal{D}\left(A^{-b}\right), \mathcal{D}\left(A^{a}\right)\right)$ is locally linearizable (see [24] pp. 91-107). Thus, we will exhibit operators $V_{0}(t), V_{1}(t)$ such that $V_{1}(t)=I-V_{0}(t)$ and

$$
\begin{aligned}
\left\|V_{0}(t)\right\|_{\mathcal{D}\left(A^{-b}\right) \rightarrow \mathcal{D}\left(A^{-b}\right)} & \leq C \\
\left\|V_{1}(t)\right\|_{\mathcal{D}\left(A^{-b}\right) \rightarrow \mathcal{D}\left(A^{a}\right)} & \leq C t^{-1} \\
\left\|V_{0}(t)\right\|_{\mathcal{D}\left(A^{a}\right) \rightarrow \mathcal{D}\left(A^{-b}\right)} & \leq C t \\
\left\|V_{1}(t)\right\|_{\mathcal{D}\left(A^{a}\right) \rightarrow \mathcal{D}\left(A^{a}\right)} & \leq C .
\end{aligned}
$$


Then

$$
K(t, x)=K\left(t, x ; \mathcal{D}\left(A^{-b}\right), \mathcal{D}\left(A^{a}\right)\right) \approx\left\|A^{-b} V_{0}(t) x\right\|+t\left\|A^{a} V_{1}(t) x\right\| .
$$

If $(a+b) \omega(A)<\pi$ we may take

$$
V_{0}(t)=t A^{a+b}\left(1+t A^{a+b}\right)^{-1}
$$

but if $(a+b) \omega(A) \geq \pi$ this is no longer a well-defined operator. We therefore define a function

$$
\psi(z)= \begin{cases}c \int_{1}^{\infty} \frac{(t z)^{a+b}}{(1+t z)^{2(a+b)}} \frac{d t}{t} & z \in \Sigma_{\phi} \\ 1 & z=0\end{cases}
$$

where

$$
c^{-1}=\int_{0}^{\infty} \frac{t^{a+b-1}}{(1+t)^{2(a+b)}} d t
$$

and $\phi>\omega(A)$ is fixed. Note that $\psi^{\prime}(z)=-c z^{a+b-1}(1+z)^{-2(a+b)}$ Let

$$
h(z)=c \int_{0}^{1} t^{a+b-1}(1+t z)^{-2(a+b)} d t .
$$

Then $h$ is analytic on the open unit disk and $h(0)=c /(a+b)$.

We then have:

$$
\begin{aligned}
\psi(z) & =z^{-(a+b)} h\left(z^{-1}\right) \quad|z|>1 \\
1-\psi(z) & =z^{a+b} h(z) \quad|z|<1 .
\end{aligned}
$$

In particular we have that $\psi(z)-(1+z)^{-1}, z^{a+b} \psi(z)-c(a+b)^{-1} z(1+z)^{-1}$ and $z^{-(a+b)}(1-\psi(z))-c(a+b)^{-1}(1+z)^{-1} \in H_{0}^{\infty}\left(\Sigma_{\phi}\right)$. If we define

$$
V_{1}(t)=\psi\left(t^{\left.\frac{1}{a+b} A\right)}\right.
$$

then it follows that (5.16) holds. Hence

$$
K(t, x) \approx\left\|A^{-b}\left(1-\psi\left(t^{\frac{1}{a+b}} A\right)\right) x\right\|+t\left\|A^{a} \psi\left(t^{\frac{1}{a+b}} A\right) x\right\| .
$$


Now let $g(z)=\psi(z)(1+z)^{a+b}$. Then

$$
\begin{aligned}
& g(z)=(1+z)^{a+b}\left(1-z^{a+b} h(z)\right)=1+O\left(|z|^{(a+b)}\right) \quad|z|<1 \\
& g(z)=(1+z)^{a+b} z^{-a-b} h\left(z^{-1}\right)=\frac{c}{a+b}+O\left(|z|^{-(a+b)}\right) \quad|z|>1 .
\end{aligned}
$$

Thus

$$
g(z)-(1+z)^{-1}-c(a+b)^{-1} z(1+z)^{-1} \in H_{0}^{\infty}\left(\Sigma_{\phi}\right)
$$

and the operators $\{g(t A): 0<t<\infty\}$ are uniformly bounded on $X$.

Similarly let $k(z)=(1+z)^{a+b} z^{-(a+b)}(1-\psi(z))$. Then

$$
\begin{aligned}
& k(z)=(1+z)^{a+b} h(z)=\frac{c}{a+b}+O\left(|z|^{(a+b)}\right) \quad|z|<1 \\
& k(z)=(1+z)^{a+b} z^{-a-b}\left(1-z^{-a-b} h\left(z^{-1}\right)=1+O\left(|z|^{-(a+b)}\right) \quad|z|>1 .\right.
\end{aligned}
$$

Thus the operators $k(t A)$ are also uniformly bounded.

Hence for $x \in X$,

$$
\begin{gathered}
\|\psi(t A) x\|=\left\|g(t A)(1+t A)^{-(a+b)} x\right\| \leq C\left\|(1+t A)^{-(a+b)} x\right\| \\
\|(1-\psi(t A)) x\|=\left\|k(t A)(t A)^{a+b}(1+t A)^{-(a+b)} x\right\| \\
\leq C\left\|(t A)^{a+b}(1+t A)^{-(a+b)} x\right\| .
\end{gathered}
$$

This leads to the upper estimate

$$
K(t, x) \leq C t^{\frac{b}{a+b}}\left\|\varphi_{a, b}\left(t^{\frac{1}{a+b}} A\right)\right\|_{X} \quad 0<t<\infty .
$$

For the converse estimate we note that

$$
\left\|t^{b} \varphi_{a, b}(t A)\right\|_{\mathcal{D}\left(A^{-b}\right) \rightarrow X},\left\|t^{-a} \varphi_{a, b}(t A)\right\|_{\mathcal{D}\left(A^{a}\right) \rightarrow X} \leq C .
$$

Theorem 5.4 Suppose $-\infty<\sigma<\tau<\infty$ and let $Y$ be an intermediate space for the Banach couple $\left(\mathcal{D}\left(A^{\sigma}\right), \mathcal{D}\left(A^{\tau}\right)\right)$. The following are equivalent:

(i) $Y=X_{E}$ for some admissible Banach function space with $-\tau<\alpha_{E} \leq \beta_{E}<-\sigma$.

(ii) $Y$ is a strict real interpolation space for the couple $\left(\mathcal{D}\left(A^{\sigma}\right), \mathcal{D}\left(A^{\tau}\right)\right)$.

Proof (i) $\Longrightarrow$ (ii). If $Y=X_{E}$ then letting $a=\tau$ and $b=-\sigma$ we have

$$
\|x\|_{Y} \approx\|\| \varphi_{a, b}(t A) x\left\|_{X}\right\|_{E} \approx\left\|t^{-b} K\left(t^{a+b}, x\right)\right\|_{E} .
$$

thus if we define the admissible function space $F$ by $h \in F$ if and only if $t^{-b} h\left(t^{a+b}\right) \in$ $E$ with the norm

$$
\|h\|_{F}=\left\|t^{-b} h\left(t^{a+b}\right)\right\|_{E}
$$


we have

$$
Y=\left(\mathcal{D}\left(A^{\sigma}\right), \mathcal{D}\left(A^{\tau}\right)\right)_{F}
$$

It is readily computed that

$$
\alpha_{F}=(a+b)^{-1}\left(\alpha_{E}+\sigma\right)>-1
$$

and

$$
\beta_{F}=(a+b)^{-1}\left(\beta_{E}+\sigma\right)<0
$$

(ii) $\Longrightarrow$ (i). If $Y=\left(\mathcal{D}\left(A^{\sigma}\right), \mathcal{D}\left(A^{\tau}\right)_{F}\right.$ we define $E=\left\{h: t^{\frac{b}{a+b}} h\left(t^{\frac{1}{a+b}}\right) \in F\right\}$ with the norm

$$
\|h\|_{E}=\left\|t^{\frac{b}{a+b}} h\left(t^{\frac{1}{a+b}}\right)\right\|_{F}
$$

and then

$$
Y=\left(\mathcal{D}\left(A^{\sigma}\right), \mathcal{D}\left(A^{\tau}\right)\right)_{F}=X_{E}
$$

and $-\tau<\alpha_{E} \leq \beta_{E} \leq-\sigma$. We omit the details.

If $E$ is an admissible Banach function space, it now follows immediately that $A$ is a sectorial operator on $X_{E}$ with domain $\left.\operatorname{Dom} A\right|_{X_{E}}=\left\{x: A x \in X_{E}\right\}$. In fact by interpolation we obtain the necessary resolvent estimates and sectoriality follows quickly from the fact that $X_{E}$ is a regular interpolation space for some pair $\left(\mathcal{D}\left(A^{\sigma}\right), \mathcal{D}\left(A^{\tau}\right)\right)$.

Theorem 5.5 Let E be an admissible Banach function space. Then A has an absolute functional calculus on $X_{E}$ of type $(c, c)$ for large enough $c>0$.

Proof Suppose $a>a^{\prime}>\max \left(0,-\alpha_{E}\right)$ and $b>b^{\prime}>\max \left(0, \beta_{E}\right)$ with $a \neq b$. We show that $A$ has a $(a+b, a+b)$-absolute functional calculus on $X_{E}$. For $s \in[1 / 2,2]$ we consider the function

$$
f_{s}(z)=\frac{1+z}{1+s z}=1+\frac{1-s}{s} \cdot \frac{s z}{1+s z} .
$$

Since $f_{S}(t A)$ is uniformly bounded for $0<t<\infty$ we have a uniform estimate

$$
\left\|\left((I+t A)(1+s t A)^{-1}\right) x\right\| \leq C\|x\| \quad 1 / 2 \leq s \leq 2,0<t<\infty .
$$

Thus we have an estimate

$$
\begin{aligned}
\left\|\varphi_{c, d}(s t A) x\right\|_{X} & =\left\|s^{c} f_{s}(t A)^{c+d} \varphi_{c, d}(t A) x\right\| \\
& \leq C\left\|\varphi_{c, d}(t A) x\right\|_{X} \quad 1 / 2 \leq s \leq 2,0<t<\infty, x \in X
\end{aligned}
$$

where $C=C(c, d, A)$. 
Now suppose $\delta>0$ and $x, y \in X_{E}$ are such that

$$
\left\|\varphi_{a+b+\delta, a+b+\delta}(s A) x\right\|_{X_{E}} \leq\left\|\varphi_{a+b, a+b}(s A) y\right\|_{X_{E}} \quad 0<s<\infty .
$$

Then for fixed $s>0$, using Theorem 5.2

$$
\begin{aligned}
& \left\|\varphi_{2 a+b+\delta, a+2 b+\delta}(s A) x\right\|_{X}\left\|\chi_{[s, 2 s)}\right\|_{E} \\
& \leq C\|\| \varphi_{a+b+\delta, a+b+\delta}(s A) \varphi_{a, b}(t A) x\left\|_{X} \chi_{[s, 2 s)}(t)\right\|_{E, t} \\
& \leq C\left\|\varphi_{a+b+\delta, a+b+\delta}(s A) \varphi_{a, b}(t A) x\right\|_{E, t} \\
& \leq C\left\|\varphi_{a+b+\delta, a+b+\delta}(s A) x\right\|_{X_{E}} \\
& \leq C\left\|\varphi_{a+b, a+b}(s A) y\right\|_{X_{E}} \\
& \leq C\left\|\varphi_{a+b, a+b}(s A) \varphi_{a+\delta, b+\delta}(t A) y\right\|_{E, t} \\
& \leq C\|\| \varphi_{a, b}(s A) \varphi_{b, a}(s A) \varphi_{a+\delta, b+\delta}(t A) y\left\|_{X}\right\|_{E, t} \\
& \leq C\|\| \min \left(\left(\frac{t}{s}\right)^{a},\left(\frac{s}{t}\right)^{b}\right) \varphi_{a, b}(s A) y\left\|_{X}\right\|_{E, t} \\
& \leq C\left\|\varphi_{a, b}(s A) y\right\|_{X} \sum_{n \in \mathbb{Z}} \min \left(2^{n a}, 2^{-n b}\right)\left\|\chi_{\left[2^{n} s, 2^{n+1} s\right)}\right\|_{E} \\
& \leq C\left\|\varphi_{a, b}(s A) y\right\|_{X}\left\|\chi_{[s, 2 s)}\right\|_{E} \sum_{n \in \mathbb{Z}} \min \left(2^{n a}, 2^{-n b}\right) \max \left(2^{-n a^{\prime}}, 2^{n b^{\prime}}\right) \\
& \leq C\left\|\varphi_{a, b}(s A) y\right\|_{X}\left\|\chi_{[s, 2 s)}\right\|_{E} .
\end{aligned}
$$

It now follows from the definition of $X_{E}$ that $\|x\|_{X_{E}} \leq C\|y\|_{X_{E}}$.

Note that if $-1<\alpha_{E} \leq \beta_{E}<0$ we deduce that $A$ has a $(a, a)$-absolute functional calculus for some $0<a<1$. Combining Theorems 5.5 and 5.4 gives us the following Corollaries.

Corollary 5.6 Suppose $-\infty<\sigma<\tau<\infty$ and let $Y$ be a strict real interpolation space for the Banach couple $\left(\mathcal{D}\left(A^{\sigma}\right), \mathcal{D}\left(A^{\tau}\right)\right)$. Then A has a $(c, c)$-absolute functional calculus on $Y$ for large enough $c>0$.

Corollary 5.7 Let $Y$ be a strict real interpolation space for the couple $(X, \mathcal{D}(A))$; then $A$ has $a(a, b)$-absolute functional calculus on $Y$ for some $0<a, b<1$, and hence has an $H^{\infty}$-functional calculus with $\omega_{H}\left(\left.A\right|_{Y}\right) \leq \omega(A)$.

If $A$ is invertible and $Y$ is given by the $(\theta, p)$-method this yields a result of Dore [14] that $A$ has the $H^{\infty}$-calculus on the spaces $(\operatorname{Dom}(A), X)_{\theta, p}$. (To be precise if $p=\infty$, one should use the inner $(\theta, \infty)$-method in order that $A$ be sectorial on the interpolation space).

Theorem 5.8 Let A be a sectorial operator on X. Then:

(i) If $X$ is a strict real interpolation space for the pair $\left(\mathcal{D}\left(A^{-b}\right), \mathcal{D}\left(A^{a}\right)\right)$ then $A$ has an $(c, c)$-absolute functional calculus for some $c<a+b$. 
(ii) If A has an $(a, b)$-absolute functional calculus then $X$ is a strict real interpolation space for the pair $\left(\mathcal{D}\left(A^{-b^{\prime}}\right), \mathcal{D}\left(A^{a^{\prime}}\right)\right)$ whenever $b^{\prime}>b$ and $a^{\prime}>a$.

(iii) If $A$ has an $(a, b)$-absolute functional calculus and $0 \neq f, g \in H^{\infty}\left(\Sigma_{\phi}\right)$ where $\phi>\omega(A)$ are such that $|f(z)|,|g(z)| \leq|z|^{a^{\prime}}(1+|z|)^{-a^{\prime}-b^{\prime}}$ for some $b^{\prime}>b, a^{\prime}>a$ then for some constant $C$,

$$
\|f(t A) x\| \leq\|g(t A) y\| \quad 0<t<\infty \Longrightarrow\|x\| \leq C\|y\| .
$$

Proof (i) This is immediate by combining Theorems 5.4 and Theorem 5.5.

We now turn to (ii) and (iii). We assume that

$$
\left\|\varphi_{a+\delta, b+\delta}(t A) x\right\| \leq\left\|\varphi_{a, b}(t A) y\right\| \quad 0<t<\infty \Longrightarrow\|x\| \leq C\|y\| .
$$

First suppose $a<a^{\prime} \leq a+\delta, b<b^{\prime} \leq b+\delta$. We use Theorem 2.4. Suppose $y \in X, x \in \mathcal{D}\left(A^{-b^{\prime}}\right)+\mathcal{D}\left(A^{a^{\prime}}\right)$ and

$$
K(t, x) \leq K(s t, y) \quad 0<t<\infty
$$

where $K(t, x)=K\left(t, x ; \mathcal{D}\left(A^{-b^{\prime}}\right), \mathcal{D}\left(A^{a^{\prime}}\right)\right)$. We will show that $x \in X$ and we have an estimate

$$
\|x\| \leq C \max \left(s^{\frac{a+b^{\prime}}{a^{\prime}+b^{\prime}}}, s^{\frac{b^{\prime}-b}{a^{\prime}+b^{\prime}}}\right)\|y\| .
$$

First we define a doubly infinite sequence $\left(\tau_{n}\right)_{n \in \mathbb{Z}}$. We may assume $y \neq 0$. We define $\tau_{n}$ for $n \leq 0$ by

$$
\tau_{n}=\sup \left\{t>0:\left\|t A(1+t A)^{-1} y\right\| \leq 2^{n-2}\|y\|\right\}
$$

Then for $n>0$ we define

$$
\tau_{n}=\inf \left\{t>0:\left\|(1+t A)^{-1} y\right\| \leq 2^{-n-1}\|y\|\right\} .
$$

It is clear that $\left(\tau_{n}\right)_{n \in \mathbb{Z}}$ is increasing. Let

$$
y_{n}=\tau_{n+1} A\left(1+\tau_{n+1} A\right)^{-1} y-\tau_{n} A\left(1+\tau_{n} A\right)^{-1} y .
$$

Then for $n \neq 0$ we have

$$
\left\|y_{n}\right\| \leq 2^{-|n|}\|y\|
$$

and hence

$$
\left\|y_{0}\right\|+\sum_{n \neq 0}\left\|y_{n}\right\| \leq 4\|y\|
$$

Thus $y=\sum_{n \in Z} y_{n}$ and convergence in $X$ is absolute. 
Since $K(s t, y) \leq \sum_{n \in \mathbb{Z}} K\left(s t, y_{n}\right)$ we can use K-divisibility (Theorem 2.2) to obtain the existence of $x_{n} \in \mathcal{D}\left(A^{-b^{\prime}}\right)+\mathcal{D}\left(A^{a^{\prime}}\right)$ so that

$$
K\left(t, x_{n}\right) \leq 6 K\left(s t, y_{n}\right), \quad n \in \mathbb{Z}
$$

and such that $x=\sum_{n \in \mathbb{Z}} x_{n}$ in $\mathcal{D}\left(A^{-b^{\prime}}\right)+\mathcal{D}\left(A^{a^{\prime}}\right)$.

Now for each $v \in \mathcal{D}\left(A^{-b^{\prime}}\right)+\mathcal{D}\left(A^{a^{\prime}}\right)$, and any $\alpha \geq a, \beta \geq b$ we have that $t \rightarrow \varphi_{\alpha, \beta}(t A) v$ defines a continuous map from $(0, \infty)$ into $X$.

Let $r=s^{\frac{1}{a^{\prime}+b^{\prime}}}$; then by Lemma 5.3 we have:

$$
\left\|\varphi_{a^{\prime}, b^{\prime}}(t A) x_{n}\right\| \leq C r^{b^{\prime}}\left\|\varphi_{a^{\prime}, b^{\prime}}(r t A) y_{n}\right\| \quad 0<t<\infty, n \in \mathbb{Z} .
$$

Then

$$
\left\|\varphi_{a+\delta, b+\delta}(t A) x_{n}\right\| \leq C r^{b^{\prime}}\left\|\varphi_{a, b}(r t A) y_{n}\right\| \quad 0<t<\infty, n \in \mathbb{Z} .
$$

Now

$$
\varphi_{a, b}(r z)=\varphi_{a, b}(z) \frac{r^{a}(1+z)^{a+b}}{(1+r z)^{a+b}} .
$$

Since $A$ admits an $H^{\infty}$-calculus (Theorem 4.2) this implies that

$$
\left\|\varphi_{a+\delta, b+\delta}(t A) x_{n}\right\| \leq C r^{b^{\prime}} \max \left(r^{a}, r^{-b}\right)\left\|\varphi_{a, b}(t A) y_{n}\right\| \quad 0<t<\infty
$$

We next check that each $x_{n} \in X$. Note that there exists a constant $c=c(a, b, \delta)$ so that

$$
x_{n}=\lim _{r \rightarrow \infty} c \int_{1 / r}^{r} \varphi_{a+\delta, b+\delta}(t A) x_{n} \frac{d t}{t}
$$

where the integrals converge in $X$ but the limit is taken with respect to the norm on $\mathcal{D}\left(A^{-b^{\prime}}\right)+\mathcal{D}\left(A^{a^{\prime}}\right)$. However we show that $t \rightarrow t^{-1} \varphi_{a+\delta, b+\delta}(t A) x_{n}$ is Bochner integrable in $X$ over $(0, \infty)$. Indeed, if $K=K(s)=C r^{b^{\prime}} \max \left(r^{a}, r^{-b}\right)$, 


$$
\begin{aligned}
\int_{0}^{\infty}\left\|\varphi_{a+\delta, b+\delta}(t A) x_{n}\right\| \frac{d t}{t} & \leq K \int_{0}^{\infty}\left\|\varphi_{a, b}(t A) y_{n}\right\| \frac{d t}{t} \\
& \leq K \int_{0}^{\infty}\left\|\varphi_{a, b}(t A) \int_{\tau_{n}}^{\tau_{n+1}} \varphi_{1,1}(u A) y \frac{d u}{u}\right\| \frac{d t}{t} \\
& \leq K \int_{0}^{\infty} \int_{\tau_{n}}^{\tau_{n+1}}\left\|\varphi_{a, b}(t A) \varphi_{\frac{1}{2}, \frac{1}{2}}(u A)\right\|\left\|\varphi_{\frac{1}{2}, \frac{1}{2}}(u A) y\right\| \frac{d u}{u} \frac{d t}{t}
\end{aligned}
$$

We now can use Lemma 3.1 to estimate

$$
\left\|\varphi_{a, b}(t A) \varphi_{\frac{1}{2}, \frac{1}{2}}(u A)\right\| \leq C \min \left((t / u)^{\theta},(u / t)^{\theta}\right)
$$

for some $\theta>0$. Hence

$$
\begin{aligned}
\int_{0}^{\infty}\left\|\varphi_{a+\delta, b+\delta}(t A) x_{n}\right\| \frac{d t}{t} & \leq C K \int_{0}^{\infty} \int_{\tau_{n}}^{\tau_{n+1}} \min \left((t / u)^{\theta},(u / t)^{\theta}\right)\left\|\varphi_{\frac{1}{2}, \frac{1}{2}}(u A) y\right\| \frac{d u}{u} \frac{d t}{t} \\
& \leq C^{\prime} K \int_{\tau_{n}}^{\tau_{n+1}}\left\|\varphi_{\frac{1}{2}, \frac{1}{2}}(u A) y\right\| \frac{d u}{u}<\infty .
\end{aligned}
$$

It follows that $x_{n} \in X$. Hence our assumptions give that

$$
\left\|x_{n}\right\| \leq C \max \left(s^{\frac{a+b^{\prime}}{a^{\prime}+b^{\prime}}}, s^{\frac{b^{\prime}-b}{a^{\prime}+b^{\prime}}}\right)\left\|y_{n}\right\|, \quad n \in \mathbb{Z} .
$$

This implies that $x=\sum_{n \in \mathbb{Z}} x_{n} \in X$ and that

$$
\|x\| \leq 4 C \max \left(s^{\frac{a+b^{\prime}}{a^{\prime}+b^{\prime}}}, s^{\frac{b^{\prime}-b}{a^{\prime}+b^{\prime}}}\right)\|y\| .
$$

Now by applying Theorems 2.4 and 5.4 one obtains $X=\left(\mathcal{D}\left(A^{-b^{\prime}}\right), \mathcal{D}\left(A^{a^{\prime}}\right)\right)_{E}$ where $-1<\alpha_{E} \leq \beta_{E}<0$. Alternatively, $X=X_{E}$ where $-a^{\prime}<\alpha_{E} \leq \beta_{E}<b^{\prime}$.

At this point (iii) is immediate by applying Theorem 5.2. Now returning to (ii) consider the case when $a^{\prime}>a+\delta$ or $b^{\prime}>b+\delta$. We can now replace $a, b$ by $a^{\prime \prime}, b^{\prime \prime}$ where $a<a^{\prime \prime}<\min \left(a+\delta, a^{\prime}\right)$ and $b<b^{\prime \prime}<\min \left(b+\delta, b^{\prime}\right)$. In view of (iii), we replace $\delta$ by a $\delta^{\prime}>0$ so that $a^{\prime}<a^{\prime \prime}+\delta^{\prime}, b^{\prime}<b^{\prime \prime}+\delta^{\prime}$ and then (ii) will follow in the general case.

Theorem 5.9 Suppose $A, B$ are two sectorial operators such that $A$ has an $(a, b)$ absolute functional calculus for some $a, b<1$. Assume $\mathcal{D}(A)=\mathcal{D}(B)$ and $\mathcal{D}\left(A^{-1}\right)=$ $\mathcal{D}\left(B^{-1}\right)$; then $B$ has an $\left(a^{\prime}, b^{\prime}\right)$-absolute functional calculus for some $a^{\prime}, b^{\prime}<1$.

Proof $X$ is a strict real interpolation space for the couple $\left(\mathcal{D}\left(A^{-1}\right), \mathcal{D}(A)\right)$. 
To conclude we discuss the results of Dore [15]. He shows that an arbitrary sectorial operator $A$ has an $H^{\infty}$-calculus on the interpolation space $(X, \operatorname{Dom}(A) \cap \operatorname{Ran}(A))_{\theta, p}$ if $0<\theta<1$ and $1 \leq p \leq \infty$. Note that $\operatorname{Dom}(A) \cap \operatorname{Ran}(A)=\mathcal{D}(A) \cap \mathcal{D}\left(A^{-1}\right)$ so this result does not follow directly from the above theorems. However, Dore's method shows that we have:

Theorem 5.10 Suppose that $Y$ is a strict interpolation space for the pair $(X, \mathcal{D}(A) \cap$ $\mathcal{R}(A))$. Then $A$ has an $H^{\infty}$-calculus on $Y$ with $\omega_{H}\left(\left.A\right|_{Y}\right) \leq \omega(A)$.

Proof The argument used by Dore is to define $B=A+A^{-1}+2 I$ and note $B$ is sectorial and invertible and $\mathcal{D}(B)=\mathcal{D}(A) \mathcal{R}(A)$. Furthermore, he shows (Theorem 2.3 from [15]) that (effectively)

$$
K(t, x ; \mathcal{D}(A) \cap \mathcal{R}(A), X) \approx K(t, x ; \mathcal{D}(A), X)+K(t, x ; \mathcal{R}(A), X) .
$$

There is a slight error in the exposition in [15] in the computation of $\|(t 1+A)(t 1+$ $b)^{-1} \|$ but is corrected by noting that

$$
(t 1+A)(t 1+B)^{-1}=1-(1+2 A)(1+A)^{-2} B(t+B)^{-1} .
$$

To complete the proof one observes that this implies that if $Y=(\mathcal{D}(A) \cap \mathcal{R}(A), X)_{E}$ then $Y=(\mathcal{D}(A), X)_{E} \cap(\mathcal{R}(A), X)_{E}$ and so since $A$ has an $H^{\infty}$-calculus on both these spaces by Corollary 5.7 it also has an $H^{\infty}$-calculus on $Y$.

We note however that this proof does not show that $A$ has an absolute functional calculus (in contrast to the situation in Corollary 5.7). This conclusion can be reached under a stronger hypothesis:

Theorem 5.11 Suppose $A$ is a sectorial operator such that $\omega(A)<\pi / 2$. If $Y$ is a strict interpolation space for the pair $(X, \mathcal{D}(A) \cap \mathcal{R}(A))$, then $A$ has an absolute functional calculus on $Y$.

To prove this theorem, we prove the following

Proposition 5.12 Suppose A is a sectorial operator such that $\omega(A)<\pi / 2$. Then

(i) $B=\frac{1}{2}\left(A+A^{-1}\right)$ is a sectorial operator and $\omega(B)<\pi / 2$.

(ii) For $0<a \leq 1$ there is a constant $C=C$ (a) so that

$$
\begin{aligned}
\frac{1}{C}\left\|\varphi_{a, a}(t B) x\right\| & \leq\left\|\varphi_{a, a}\left(s_{1} A\right) x\right\|+\left\|\varphi_{a, a}\left(s_{2} A\right) x\right\| \\
& \leq C\left\|\varphi_{a, a}(t B) x\right\| \quad x \in X, 0<t \leq 1,
\end{aligned}
$$

where $s_{1}, s_{2}$ are the roots of $t z^{2}-2 z+t=0$.

Proof (i) Suppose $\Re \lambda<0$. Then

$$
(1-\lambda B)^{-1}=-\frac{2}{\lambda} A\left(1-\zeta_{1} A\right)^{-1}\left(1-\zeta_{2} A\right)^{-1}
$$


where $\zeta_{1}, \zeta_{2}$ are the roots of $\lambda z^{2}-2 z+\lambda=0$. Note that if $z+z^{-1}=2 / \lambda$ then $\Re z<0$ so that $\Re \zeta_{1}, \Re \zeta_{2}<0$.

$$
\left\|(1-\lambda B)^{-1}\right\| \leq C|\lambda|^{-1} \min \left(\left|\zeta_{1}\right|^{-1},\left|\zeta_{2}\right|^{-1}\right)
$$

for a suitable constant $C$. Since $\left|\zeta_{1}\right|+\left|\zeta_{2}\right| \geq 2 / \lambda$ we conclude that

$$
\left\|(1-\lambda B)^{-1}\right\| \leq C^{\prime} \quad \mathfrak{R} \lambda<0
$$

and $B$ is sectorial with $\omega(B)<\pi / 2$.

(ii) Let us first treat the case $a=1$. We may suppose that $0<s_{1} \leq 1 \leq s_{2}<\infty$. By the argument of (i) we have

$$
\begin{aligned}
(1+t B)^{-1} & =\frac{2}{t} A\left(1+s_{1} A\right)^{-1}\left(1+s_{2} A\right)^{-1} . \\
B(1+t B)^{-2} & =\frac{2}{t^{2}} A\left(1+A^{2}\right)\left(1+s_{1} A\right)^{-2}\left(1+s_{2} A\right)^{-2} .
\end{aligned}
$$

Thus

$$
\varphi_{1,1}\left(s_{1} A\right)=\frac{t s_{1}}{2}\left(1+s_{2} A\right)^{2}\left(1+A^{2}\right)^{-1} \varphi_{1,1}(t B) .
$$

Now since $0<t \leq 1$ we may put $s_{1}=1 / s_{2}$ and $t s_{2} \leq 2$ and note that $\left\|\left(1+s_{2} A\right)^{2}\left(1+A^{2}\right)^{-1}\right\| \leq s_{2}^{2}\left(\left\|\left(1+A^{2}\right)^{-1}\right\|+2\left\|A\left(1+A^{2}\right)^{-1}\right\|+\left\|A^{2}\left(1+A^{2}\right)^{-1}\right\|\right)$ so that we have an estimate

$$
\left\|\varphi_{1,1}\left(s_{1} A\right) x\right\| \leq C_{1}\left\|\varphi_{1,1}(t B) x\right\| \quad x \in X, 0<t \leq 1 .
$$

On other hand

$$
\varphi_{1,1}\left(s_{2} A\right)=\frac{t s_{2}}{2}\left(1+s_{1} A\right)^{2}\left(1+A^{2}\right)^{-1} \varphi_{1,1}(t B) .
$$

This time we observe that $t s_{1} \leq 1$ and that

$$
\left\|\left(1+s_{1} A\right)^{2}\left(1+A^{2}\right)^{-1}\right\| \leq C^{\prime}
$$

for a suitable constant $C^{\prime}$ since $0<s_{1} \leq 1$. Thus

$$
\left\|\varphi_{1,1}\left(s_{2} A\right) x\right\| \leq C_{2}\left\|\varphi_{1,1}(t B) x\right\| \quad x \in X, 0<t \leq 1
$$

Combining these gives the left hand estimate in (ii) when $a=1$. 
To obtain the right-hand estimate (for $a=1$ ) we note that

$$
\varphi_{1,1}\left(s_{1} A\right)+\varphi_{1,1}\left(s_{2} A\right)=\left(t B+t^{2}\right)(1+t B)^{-2} .
$$

Now $B$ is invertible and so

$$
\varphi_{1,1}\left(s_{1} A\right)+\varphi_{1,1}\left(s_{2} A\right)=\left(1+t B^{-1}\right) \varphi_{1,1}(t B)
$$

which gives the right hand estimate.

To extend these results to $a<1$ we let $B_{a}=\frac{1}{2}\left(A^{a}+A^{-a}\right)$ when $-1<a<1$. We observe that $f(A)$ is a bounded invertible operator where

$$
f(z)=\frac{\left(z+z^{-1}\right)^{a}}{z^{a}+z^{-a}} .
$$

Here $1-f(z)$ and $1-1 / f(z)$ belong to $H_{0}^{\infty}\left(\Sigma_{\phi}\right)$ as long as $\phi<\pi / 2$. It follows that $B^{a}=2^{a-1} f(A) B_{a}$ and $B^{-a}=2^{1-a} f(A)^{-1}\left(B_{a}\right)^{-1}$ and in particular $\mathcal{D}\left(B^{a}\right)=\mathcal{D}\left(B_{a}\right), \mathcal{D}\left(B^{-a}\right)=\mathcal{D}\left(B_{a}^{-1}\right)$. We can then use Proposition 5.3 to deduce that

$$
\left\|\varphi_{1,1}\left(t B^{a}\right) x\right\| \approx\left\|\varphi_{1,1}\left(t B_{a}\right) x\right\| \quad x \in X, 0<t<\infty .
$$

Here we use $\approx$ to mean the existence of a constant $C$ independent of $t, x$ (but depending on $a$ ) so that

$$
C^{-1}\left\|\varphi_{1,1}\left(t B^{a}\right) x\right\| \leq\left\|\varphi_{1,1}\left(t B_{a}\right) x\right\| \leq C\left\|\varphi_{1,1}\left(t B^{a}\right) x\right\| \quad 0<t<\infty .
$$

Now suppose $0<t \leq 1$ and $s_{1}=s_{1}(t), s_{2}=s_{2}(t)$ are the roots of $t z^{2}-2 z+t=0$ as above. We have

$$
\begin{aligned}
\left\|\varphi_{a, a}\left(s_{1} A\right) x\right\|+\left\|\varphi_{a, a}\left(s_{2} A\right) x\right\| & \approx\left\|\varphi_{1,1}\left(s_{1}^{a} A^{a}\right) x\right\|+\left\|\varphi_{1,1}\left(s_{2}^{a} A^{a}\right) x\right\| \\
& \approx\left\|\varphi_{1,1}\left(2\left(s_{1}^{a}+s_{2}^{a}\right)^{-1} B_{a}\right) x\right\| \\
& \approx\left\|\varphi_{1,1}\left(2^{a}\left(s_{1}^{a}+s_{2}^{a}\right)^{-1} B^{a}\right) x\right\| \\
& \approx\left\|\varphi_{a, a}\left(2\left(s_{1}^{a}+s_{2}^{a}\right)^{-1 / a} B\right) x\right\| \\
& \approx\left\|\varphi_{a, a}(t B) x\right\|
\end{aligned}
$$

since

$$
2\left(s_{1}^{a}+s_{2}^{a}\right)^{-1 / a} \approx t
$$


Proof of Theorem 5.11 It follows from Theorem 5.8 (i) that $B=\frac{1}{2}\left(A+A^{-1}\right)$ has an $(a, a)$-absolute functional calculus on $Y$ for some $a<1$. Thus using (iii) of Theorem 5.8 and Proposition 4.1 we have that

$\left\|\varphi_{1,1}(t B) y_{1}\right\|_{Y} \leq\left\|\varphi_{a, a}(t B) y_{2}\right\|_{Y} \quad 0<t \leq 1 \Longrightarrow\left\|y_{1}\right\|_{Y} \leq C\left\|y_{2}\right\|_{Y} \quad y_{1}, y_{2} \in Y$.

Now suppose

$$
\left\|\varphi_{1,1}(t A) y_{1}\right\|_{Y} \leq\left\|\varphi_{a, a}(t A) y_{2}\right\|_{Y} \quad 0<t<\infty
$$

Then by Proposition 5.12 we deduce that for a suitable constant $C_{1}$ we have

$$
\left\|\varphi_{1,1}(t B) y_{1}\right\|_{Y} \leq C_{1}\left\|\varphi_{a, a}(t B) y_{2}\right\|_{Y} \quad 0<t \leq 1
$$

and

$$
\left\|y_{1}\right\|_{Y} \leq C C_{1}\left\|y_{2}\right\|_{Y}
$$

\section{The vector-valued functional calculus for operators with an absolute functional calculus}

We next discuss the vector-valued functional calculus for a sectorial operator with an absolute functional calculus. We will first state a result which indicates that when $A$ has an absolute functional calculus then the main theorems of [18] hold without R-boundedness assumptions. Later, we will need a more delicate form of this result, and for this we will supply a detailed proof. Recall that $\mathcal{A}$ is the algebra of all operators on $X$ with commute with the resolvent operators $R(\lambda, A)$ for $\lambda$ in the resolvent set of $A$.

Theorem 6.1 Suppose A is a sectorial operator with an absolute functional calculus and $F \in H^{\infty}\left(\Sigma_{\phi}, \mathcal{A}\right)$ for some $\phi>\omega$. Then $F(A)$ is a bounded operator on $X$.

Proof The proof of this theorem is identical to the proof of the corresponding scalar result, Theorem 4.2 and we therefore omit it.

This leads to some standard applications which are quite routine.

Corollary 6.2 Suppose $A$ and $B$ are commuting sectorial operators and A has absolute calculus. Suppose further that $f \in H^{\infty}\left(\Sigma_{\phi} \times \Sigma_{\rho}\right)$ where $\phi>\omega(A), \psi>\omega(B)$ is such that $f(z, B)$ is a bounded operator for every $z \in \Sigma_{\phi}$ and the family $\{f(z, B)$ : $\left.z \in \Sigma_{\phi}\right\}$ is uniformly bounded. Then $f(A, B)$ is a bounded operator.

Proof Consider $F(z)=f(z, B)$. Using the integral representation of $f(z, B)$ from Sect. 2 and commutativity of $A$ and $B$, we conclude that $F(z) \in H^{\infty}\left(\Sigma_{\phi}, \mathcal{A}\right)$. It follows from Theorem 6.1 that $F(A)$ is a bounded operator. 
Theorem 6.3 Let $A$ and $B$ are commuting sectorial operators so that $\omega(A)+\omega(B)<$ $\pi$. Suppose A has absolute calculus. Then $A+B$ is closed on the domain $\operatorname{Dom}(A) \cap$ $\operatorname{Dom}(B)$ and there is a constant $C$ such that

$$
\|A x\|+\|B x\| \leq C\|A x+B x\|, \quad x \in \mathcal{D}(A) \cap \mathcal{D}(B)
$$

and $(A+B)$ is invertible if either $A$ or $B$ is invertible.

Proof Choose $\phi, \phi^{\prime}$ with $\omega(A)<\phi, \omega(B)<\phi^{\prime}$ and $\phi+\phi^{\prime}<\pi$. The function $f(z, w)=z(z+w)^{-1}$ is in $H^{\infty}\left(\Sigma_{\phi} \times \Sigma_{\phi^{\prime}}\right)$ and the family $f(z, B)=-z R(-z, B)$ for $z \in \Sigma_{\phi^{\prime}}$ is uniformly bounded. By Corollary 6.2 we obtain that $f(A, B)$ is a bounded operator. It was shown in $[18,19]$ that this implies the norm estimate 6.20 and hence that $A+B$ is closed.

Now suppose that $A$ is invertible. Then since $A(A+B)^{-1}$ is bounded, we conclude that also $(A+B)$ is invertible. In the case $B$ is invertible we write $I=A(A+B)^{-1}+$ $B(A+B)^{-1}$ and see that $B(A+B)^{-1}$ is bounded and thus $(A+B)$ invertible.

The following theorem is the analogue of the result of [19]. We omit the proof which is similar to the previous theorem.

Theorem 6.4 Suppose A and B are commuting sectorial operators each with an $H^{\infty}$ calculus and assume $A$ has an absolute functional calculus. Then $(A, B)$ has a joint $H^{\infty}$-functional calculus. More precisely if $\phi>\omega_{H}(A)$ and $\psi>\omega_{H}(B)$ then for every $f \in H^{\infty}\left(\Sigma_{\phi} \times \Sigma_{\psi}\right), f(A, B)$ is a bounded operator and we have an estimate

$$
\|f(A, B)\| \leq C\|f\|_{H^{\infty}\left(\Sigma_{\phi} \times \Sigma_{\psi}\right)} .
$$

\section{Mild solutions and well-posed equations}

Let us assume that $A$ is a sectorial operator on $X$ and that $B$ is a closed operator with non-empty resolvent set. Suppose $A$ and $B$ are resolvent commuting. Then the operator $A+B$ with domain $\operatorname{Dom}(A) \cap \operatorname{Dom}(B)$ may not be closed. $A+B$ may however be extended to a closed operator on the domain

$$
\begin{gathered}
\operatorname{Dom}(A+B)=\left\{x \in X: \exists x_{n} \in \operatorname{Dom}(A) \cap \operatorname{Dom}(B), \lim _{n \rightarrow \infty} x_{n}=x,\right. \\
\left.\exists \lim _{n \rightarrow \infty} A x_{n}+B x_{n}:=(A+B) x\right\} .
\end{gathered}
$$

To see this we need only check that if $x_{n} \in \operatorname{Dom}(A) \cap \operatorname{Dom}(B)$ and $\lim _{n \rightarrow \infty} x_{n}=0$ and $\lim _{n \rightarrow \infty}\left(A x_{n}+B x_{n}\right)=y$ then $y=0$. But $\lim _{n \rightarrow \infty} A(1+A)^{-2} x_{n}=0$ and $\lim _{n \rightarrow \infty} A^{2}(1+A)^{-2} x_{n}=0$. Hence $\lim _{n \rightarrow \infty} B A(1+A)^{-2} x_{n}=A(1+A)^{-2} y$. Since $B$ is closed $A(1+A)^{-2} y=0$ and thus $y=0$. Note that if $x \in \operatorname{Dom}(A+B)$ then $v_{n}(A) x \in \operatorname{Dom}(A) \cap \operatorname{Dom}(B)$ for all $n$ and $\sup \left\|A v_{n}(A) x+B v_{n}(A) x\right\|<\infty$.

In this section, we consider two questions concerning the equation (for fixed $y \in X$ )

$$
(A+B) x=y
$$


We say that a solution $x$ of (7.21) is mild solution if $x \in \operatorname{Dom}(A+B)$ and a strong or classical solution if $x \in \operatorname{Dom}(A) \cap \operatorname{Dom}(B)$.

The closed operator $(A+B, \operatorname{Dom}(A+B))$ is invertible if and only if for every $y \in X$ there is a unique mild solution of (7.21). On other hand (7.21) is said to be well-posed if every mild solution is a strong solution. (7.21) is well-posed if and only if there is a constant $C$ so that

$$
\|A x\| \leq C\|A x+B x\| \quad x \in \operatorname{Dom}(A) \cap \operatorname{Dom}(B)
$$

and this is equivalent to the requirement that $\operatorname{Dom}(A+B)=\operatorname{Dom}(A) \cap \operatorname{Dom}(B)$.

Let us first investigate conditions for the homogeneous equation

$$
A x+B x=0, \quad x \in \operatorname{Dom}(A+B)
$$

to have a unique solution.

Proposition 7.1 The following conditions imply that (7.22) has a unique solution.

(i) There exists a constant $C$ so that for every $\lambda \in \mathbb{C}$ we have either $\lambda \notin \operatorname{Sp}(A)$ and $\left\|(\lambda-A)^{-1}\right\| \leq C$ or $-\lambda \notin S p(B)$ and $\left\|(\lambda+B)^{-1}\right\| \leq C$.

(ii) There exists a constant $C$ so that for every $\lambda \in \mathbb{C} \backslash\{0\}$ we have either $\lambda \notin \operatorname{Sp}(A)$ and $\left\|\lambda(\lambda-A)^{-1}\right\| \leq C$ or $-\lambda \notin S p(B)$ and $\left\|\lambda(\lambda+B)^{-1}\right\| \leq C$.

Proof (i) Suppose $x \in \operatorname{Dom}(A) \cap \operatorname{Dom}(B)$ and $A x+B x=0$. Consider the analytic functions $F(\lambda)=(\lambda-A)^{-1}$ defined on $\mathbb{C} \backslash \operatorname{Sp}(A)$ and $G(\lambda)=(\lambda+B)^{-1}$ defined on $\mathbb{C} \backslash \operatorname{Sp}(-B)$. If $\lambda \notin \operatorname{Sp}(A) \cup \operatorname{Sp}(-B)$ then

$$
\begin{aligned}
x & =(\lambda-A)(\lambda-A)^{-1} x \\
& =\lambda(\lambda-A)^{-1} x-(\lambda-A)^{-1} A x \\
& =\lambda(\lambda-A)^{-1} x+(\lambda-A)^{-1} B x \\
& =\lambda(\lambda-A)^{-1} x+B(\lambda-A)^{-1} x \\
& =(\lambda+B)(\lambda-A)^{-1} x
\end{aligned}
$$

so that $F(\lambda) x=G(\lambda) x$. Hence $F$ extends to a bounded entire function and Liouville's theorem implies that $F$ vanishes identically.

Now suppose $x \in \operatorname{Dom}(A+B)$ and $(A+B) x=0$. Then $v_{n}(A) x \in \operatorname{Dom}(A) \cap$ $\operatorname{Dom}(B)$ for all $n$ and $(A+B) v_{n}(A) x=0$. Thus $v_{n}(A) x=0$ and so $x=0$.

(ii) is similar.

We shall define a contour in $\mathbb{C}$ to be acceptable if it can be decomposed into a finite union of curves $\Gamma_{j}$ each of which may be parameterized by a Lipschitz map $t \rightarrow z(t)$ for $a<t<b$ where $-\infty \leq a<b \leq \infty$ and such for some constant $C$ we have $C^{-1}|t| \leq|z(t)| \leq C|t|$. The main application use of this condition is that we will have a estimate 


$$
\int_{\Gamma} \min \left(|t \zeta|^{\delta},|t \zeta|^{-\delta}\right) \frac{|d \zeta|}{|\zeta|} \leq C \quad t>0
$$

where $C=C(\delta)$ is independent of $t$.

Theorem 7.2 Let A be a sectorial operator and suppose B is a closed operator. Suppose the homogeneous equation (7.22) has a unique solution. Suppose $\omega(A)<\phi<$ $\psi<\pi$. Suppose $\Gamma$ is an acceptable contour contained in $\Sigma_{\phi} \cup\{0\}$ and with the following properties:

$$
\begin{aligned}
& \sup _{\lambda \in \Gamma \backslash\{0\}} \min (1,|\lambda|)\left\|(\lambda-A)^{-1}\right\|<\infty . \\
& \sup _{\lambda \in \Gamma}\left\|(\lambda+B)^{-1}\right\|<\infty . \\
& \int_{\Gamma} f(\zeta)(\zeta-A)^{-1} d \zeta=f(A)
\end{aligned}
$$

if $f, z f \in H_{0}^{\infty}\left(\Sigma_{\psi}\right)$.

$$
\int_{\Gamma} f(\zeta)(\zeta+B)^{-1} d \zeta=0
$$

if $f, z f \in H_{0}^{\infty}\left(\Sigma_{\phi}\right)$. Then each of the following conditions suffices for $(A+B$, $\operatorname{Dom}(A+B))$ to be invertible:

(i)

$$
\int_{\Gamma}\left\|(\zeta-A)^{-1}\right\|\left\|(\zeta+B)^{-1}\right\||d \zeta|<\infty
$$

or

(ii) A has an absolute functional calculus and for some constant $C$,

$$
\left\|(\lambda-A)^{-1}\right\|\left\|(\lambda+B)^{-1}\right\| \leq C|\lambda|^{-1} \quad \lambda \in \Gamma .
$$

If in (ii) we also have for some constant $C^{\prime}$,

$$
\max \left(\left\|(\lambda-A)^{-1}\right\|,\left\|(\lambda+B)^{-1}\right\|\right) \leq C^{\prime}|\lambda|^{-1} \quad \lambda \in \Gamma
$$

then $A x+B x=y$ is well-posed.

Proof Before showing the details of the proof, we pause to understand the situation graphically (Fig. 1). 


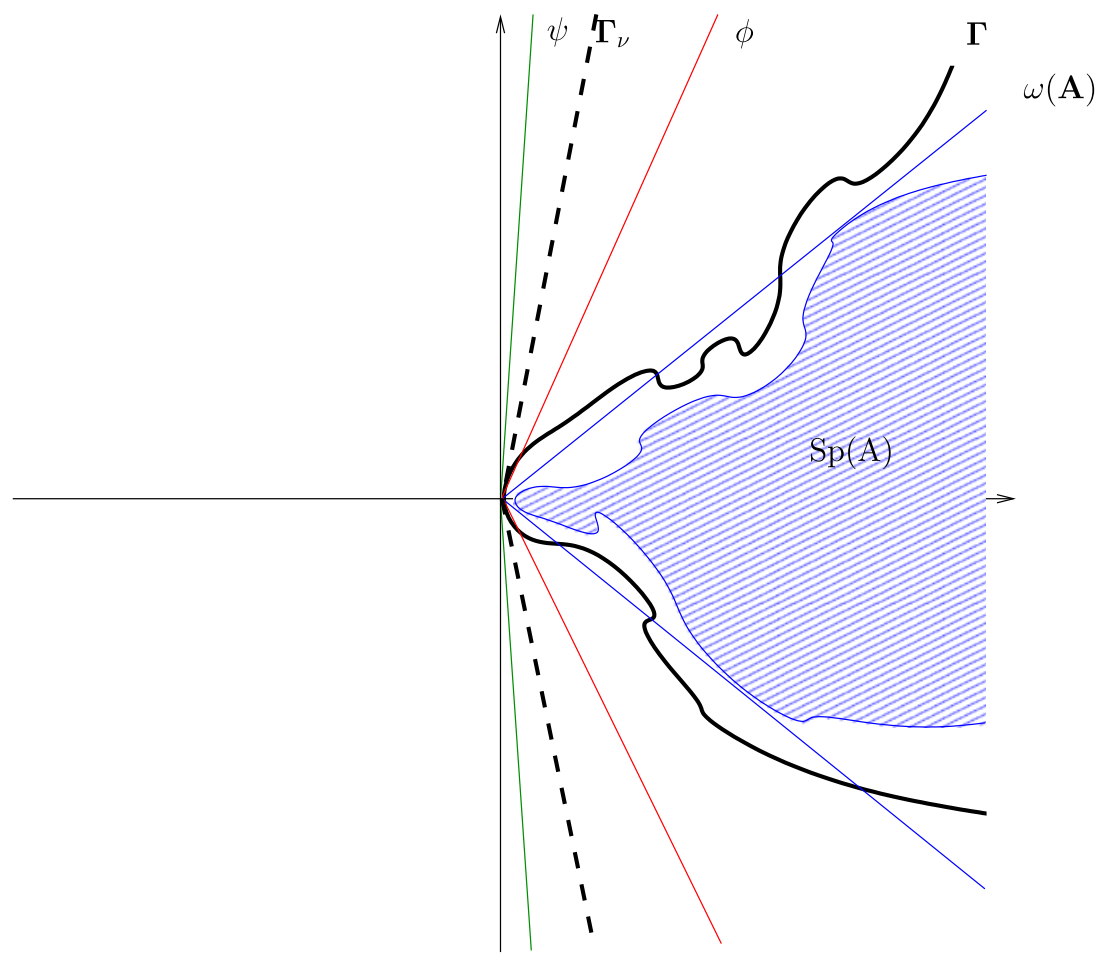

Fig. 1 This figure illustrates Theorem 7.2

First observe that, by our definition of an acceptable contour, under (7.23) and (7.24) the conditions that $f, z f \in H_{0}^{\infty}\left(\Sigma_{\psi}\right)$ implies that the integrals in (7.25) and (7.26) exist as Bochner integrals and so does

$$
\mathcal{T}(f)=\frac{1}{2 \pi i} \int_{\Gamma} f(\zeta)(\zeta+B)^{-1}(\zeta-A)^{-1} d \zeta
$$

Claim 1 If $f, z^{-1} f, g, z g \in H_{0}^{\infty}\left(\Sigma_{\psi}\right)$ then

$$
\mathcal{T}(f g)=f(A) \mathcal{T}(g) .
$$

Proof of Claim 1 Suppose $\phi<v<\psi$. Then

$$
f(A)=\frac{1}{2 \pi i} \int_{\Gamma_{v}} f(\lambda)(\lambda-A)^{-1} d \lambda
$$


and so

$$
f(A) \mathcal{T}(g)=-\frac{1}{4 \pi^{2}} \int_{\Gamma_{\nu}} \int_{\Gamma} f(\lambda) g(\zeta)(\zeta+B)^{-1}(\lambda-A)^{-1}(\zeta-A)^{-1} d \zeta d \lambda
$$

as a Bochner integral.

By the resolvent equation

$$
(\lambda-A)^{-1}(\zeta-A)^{-1}=\frac{1}{\zeta-\lambda}\left((\lambda-A)^{-1}-(\zeta-A)^{-1}\right) .
$$

We now observe that for $\lambda \in \Gamma_{\nu}$ and $\zeta \in \Gamma$ we have $|\lambda-\zeta| \geq c|\lambda|$ where $c=$ $\sin (v-\phi)>0$. Since

$$
\int_{\Gamma_{v}}|f(\lambda)| \frac{|d \lambda|}{|\lambda|}<\infty
$$

the integrals

$$
\int_{\Gamma_{v}} \int_{\Gamma} \frac{f(\lambda)}{\zeta-\lambda} g(\zeta)(\zeta+B)^{-1}(\lambda-A)^{-1} d \zeta d \lambda
$$

and

$$
\int_{\Gamma_{\nu}} \int_{\Gamma} \frac{f(\lambda)}{\zeta-\lambda} g(\zeta)(\zeta+B)^{-1}(\zeta-A)^{-1} d \zeta d \lambda
$$

both converge as Bochner integrals. The first integral vanishes by using (7.26) (integrating first with respect to $\zeta$ ). Thus

$$
\begin{aligned}
f(A) \mathcal{T}(g) & =\frac{1}{4 \pi^{2}} \int_{\Gamma_{\nu}} \int_{\Gamma} \frac{f(\lambda)}{\zeta-\lambda} g(\zeta)(\zeta+B)^{-1}(\zeta-A)^{-1} d \zeta d \lambda \\
& =\frac{1}{2 \pi i} \int_{\Gamma} f(\zeta) g(\zeta)(\zeta+B)^{-1}(\zeta-A)^{-1} d \zeta \\
& =\mathcal{T}(f g) .
\end{aligned}
$$

This completes the proof of claim 1 .

Now suppose $z f, z^{-1} f \in H_{0}^{\infty}\left(\Sigma_{\psi}\right)$. Then by claim 1 we have

$$
\mathcal{T}\left(z f^{2}\right)=A f(A) \mathcal{T}(f)=A \mathcal{T}\left(f^{2}\right)
$$


and so $\mathcal{T}\left(f^{2}\right)$ has range in $\operatorname{Dom}(A)$. Now

$$
f^{2}(A)=\frac{1}{2 \pi i} \int_{\Gamma} f(\zeta)^{2}(\zeta-A)^{-1} d \zeta
$$

and

$$
\mathcal{T}\left(z f^{2}\right)=\frac{1}{2 \pi i} \int_{\Gamma} \zeta f(\zeta)^{2}(\zeta+B)^{-1}(\zeta-A)^{-1} d \zeta
$$

both as Bochner integrals. Hence

$$
f^{2}(A)-\mathcal{T}\left(z f^{2}\right)=\frac{1}{2 \pi i} \int_{\Gamma} f(\zeta)^{2} B(\zeta+B)^{-1}(\zeta-A)^{-1} d \zeta
$$

as a Bochner integral. Since $B$ is closed this implies that for every $x \in X$,

$$
f^{2}(A) x-\mathcal{T}\left(z f^{2}\right) x=B\left(\frac{1}{2 \pi i} \int_{\Gamma} f(\zeta)^{2}(\zeta+B)^{-1}(\zeta-A)^{-1} x d \zeta\right)
$$

and the right-integral is in $\operatorname{Dom}(B)$ i.e., $\mathcal{T}\left(f^{2}\right) x \in \operatorname{Dom}(B)$ and

$$
f^{2}(A) x=A \mathcal{T}\left(f^{2}\right) x+B \mathcal{T}\left(f^{2}\right) x .
$$

Since $A+B$ is one-one to prove that $(A+B)^{-1}$ is bounded it therefore suffices to show an estimate

$$
\left\|\mathcal{T}\left(f^{2}\right)\right\| \leq C\left\|f^{2}\right\|_{H^{\infty}\left(\Sigma_{\psi}\right)}
$$

for all functions $f$ such that $z f, z^{-1} f \in H_{0}^{\infty}\left(\Sigma_{\psi}\right)$. Indeed suppose we have such an estimate and $x \in \operatorname{Ran} f^{2}(A)$. Then $x=f^{2}(A) y=(A+B) \mathcal{T}\left(f^{2}\right) y$ for some $y \in X$ and thus $x \in \operatorname{Dom}(A+B)^{-1}$. Since $z v_{n}^{2}(z), z^{-1} v_{n}^{2}(z) \in H_{0}^{\infty}\left(\Sigma_{\psi}\right)$ we also get $v_{n}^{4}(A) x \in \operatorname{Dom}(A+B)^{-1}$ and

$$
\lim _{n \rightarrow \infty}(A+B)^{-1}\left(x-v_{n}(A)^{4} x\right)=\lim _{n \rightarrow \infty}\left(\mathcal{T}\left(f^{2}\right) y-\mathcal{T}\left(f^{2}\right) v_{n}^{4} y\right)=0 .
$$

Thus

$$
\left\|(A+B)^{-1} x\right\| \leq \limsup _{n \rightarrow \infty}\left\|(A+B)^{-1} v_{n}^{4}(A) x\right\| \leq \limsup _{n \rightarrow \infty}\left\|\mathcal{T}\left(v_{n}^{2}\right) x\right\| \leq C\|x\| .
$$

Now, our claim follows from choosing a function $f$ such that $\operatorname{Ran} f^{2}(A)$ is dense in $X$, e.g., $f(z)=v_{2}^{2}(z)$.

In case (i) this is a trivial norm estimate. We turn to case (ii). 
Assume as before $z f, z^{-1} f \in H_{0}^{\infty}\left(\Sigma_{\psi}\right)$. Assume that $g \in H_{0}^{\infty}\left(\Sigma_{\phi^{\prime}}\right)$ for some $\phi^{\prime}>\omega(A)$ and that $0<\delta<\frac{1}{2}$ are such that

$$
\left\|\varphi_{\delta, \delta}(t A) g(t A) x\right\| \leq\|g(t A) y\| \quad 0<t<\infty \Longrightarrow\|x\| \leq C\|y\| .
$$

Now suppose $x \in \operatorname{Dom}\left(A^{\frac{1}{2}}\right)$. Let $f^{2}=\varphi_{2,1} f_{0}$ where $f_{0}, z f_{0} \in H_{0}^{\infty}\left(\Sigma_{\psi}\right)$. Then

$$
\begin{aligned}
\mathcal{T}\left(z^{-\frac{1}{2}} f^{2}\right)\left(A^{1 / 2} x\right) & =\frac{1}{2 \pi i} \int_{\Gamma} \zeta^{-\frac{1}{2}} f^{2}(\zeta)(\zeta+B)^{-1}(\zeta-A)^{-1} A^{1 / 2} x d \zeta \\
& =\frac{1}{2 \pi i} \int_{\Gamma} \varphi_{\frac{3}{2}, \frac{3}{2}}(\zeta) f_{0}(\zeta)(\zeta+B)^{-1}(\zeta-A)^{-1} A^{1 / 2} x d \zeta \\
& =\varphi_{\frac{3}{2}, \frac{3}{2}}(A) \int_{\Gamma} f_{0}(\zeta)(\zeta+B)^{-1}(\zeta-A)^{-1} A^{1 / 2} x d \zeta \\
& =\varphi_{2,1}(A) \int_{\Gamma} f_{0}(\zeta)(\zeta+B)^{-1}(\zeta-A)^{-1} x d \zeta \\
& =\varphi_{2,1}(A) \mathcal{T}\left(f_{0}\right) x \\
& =\mathcal{T}\left(f^{2}\right) x
\end{aligned}
$$

Here we applied claim 1 again since $\varphi_{\frac{3}{2}}, \frac{3}{2}, z^{-1} \varphi_{\frac{3}{2}, \frac{3}{2}}, f_{0}, z f_{0} \in H_{0}^{\infty}\left(\Sigma_{\psi}\right)$.

We have

$$
A^{\frac{1}{2}}(\zeta-A)^{-1}=|\zeta|^{-\frac{1}{2}} \varphi_{\frac{1}{2}, \frac{1}{2}}\left(|\zeta|^{-1} A\right)\left((|\zeta|+\zeta)(\zeta-A)^{-1}-I\right)
$$

so that we have an estimate

$$
\begin{gathered}
\left\|\varphi_{\delta, \delta}(t A) A^{\frac{1}{2}}(\zeta-A)^{-1}\right\| \leq C|\zeta|^{-\frac{1}{2}} \min \left(|t \zeta|^{\delta},|t \zeta|^{-\delta}\right)\left(\left\|\zeta(\zeta-A)^{-1}\right\|+1\right) . \\
\text { If } t>0 \text { and } y=\mathcal{T}\left(f^{2}\right) x \text { then } \\
\varphi_{\delta, \delta}(t A) g(t A) y=\frac{1}{2 \pi i} \int_{\Gamma} \zeta^{-\frac{1}{2}} f^{2}(\zeta)(\zeta+B)^{-1} \varphi_{\delta, \delta}(t A) A^{\frac{1}{2}}(\zeta-A)^{-1} g(t A) x d \zeta .
\end{gathered}
$$

Thus

$$
\begin{aligned}
\left\|\varphi_{\delta, \delta}(t A) g(t A) y\right\| \leq & C\left\|f^{2}\right\|_{H^{\infty}\left(\Sigma_{\psi}\right)}\|g(t A) x\| \int_{\Gamma} \min \left(|t \zeta|^{\delta},|t \zeta|^{-\delta}\right) \\
& \left.\times\left(1+|\zeta|\left\|(\zeta-A)^{-1}\right\|\right)\left\|(\zeta+B)^{-1}\right\|\right) \frac{d|\zeta|}{|\zeta|} \\
\leq & C\left\|f^{2}\right\|_{H^{\infty}\left(\Sigma_{\psi}\right)}\|g(t A) x\|,
\end{aligned}
$$


using the fact that $\Gamma$ is an acceptable contour and (7.28). Thus

$$
\|y\| \leq C\left\|f^{2}\right\|_{H^{\infty}\left(\Sigma_{\psi}\right)}\|x\| .
$$

This completes the proof of (ii).

For the last part we observe that $A \mathcal{T}\left(f^{2}\right)=\mathcal{T}\left(z f^{2}\right)$ and use a similar argument to give an estimate

$$
\|A y\| \leq C\left\|f^{2}\right\|_{H^{\infty}\left(\Sigma_{\psi}\right)}\|x\|
$$

We now specialize to the case when $-A$ is the generator of a bounded semigroup $\left\{e^{-t A}: t>0\right\}$. In this case we have an estimate

$$
\left\|(\lambda-A)^{-1}\right\| \leq C|\Re \lambda|^{-1} \quad \Re \lambda<0 .
$$

Theorem 7.3 Suppose $A$ is a sectorial operator such that $-A$ is the generator of a bounded semigroup. Suppose B is a closed operator. Assume further that $\operatorname{Sp}(B) \subset$ $\{z: \Re z>0\}$ and

$$
\sup _{\Re \lambda \geq 0}\left\|(\lambda+B)^{-1}\right\|<\infty
$$

Then each of the following conditions implies that the equation $A x+B x=y$ admits a unique mild solution:

(i) A is invertible and

$$
\int_{-\infty}^{\infty}\left\|(i t+B)^{-1}\right\|^{2} d t<\infty .
$$

(ii) A has an absolute functional calculus and for some $\phi<\pi / 2$ we have $\operatorname{Sp}(B) \subset$ $\Sigma_{\phi}$ and

$$
\sup _{\lambda \notin \Sigma_{\phi}}\left\|(\lambda+B)^{-1}\right\|<\infty
$$

(iii) A has an absolute functional calculus and

$$
\sup _{-\infty<t<\infty}|t|^{\frac{1}{2}}\left\|(i t+B)^{-1}\right\|<\infty .
$$

Proof First we note that from the initial hypotheses there exists $\alpha>0$ so that

$$
\sup _{\Re \lambda>-\alpha}\left\|(\lambda+B)^{-1}\right\|<\infty
$$


and so the conditions of Proposition 7.1 (i) are satisfied. Thus $(A+B)$ is injective on its domain.

Let us prove (iii) first. For this we note that if $t \in \mathbb{R}$ and $|s|<\frac{1}{2}\left\|(i t+B)^{-1}\right\|^{-1}$, $(i t+s+B)$ is invertible

$$
\left\|(i t+s+B)^{-1}\right\| \leq 2\left\|(i t+B)^{-1}\right\| .
$$

Since the function $t \mapsto\left\|(-i t+B)^{-1}\right\|^{-1}$ is Lipschitz we can define an acceptable contour by

$$
\zeta(t)= \begin{cases}-i t-\frac{1}{4}\left\|(-i t+B)^{-1}\right\|^{-1} & |t|>1 \\ -i t-\frac{|t|}{4}\left\|(-i t+B)^{-1}\right\|^{-1} & |t| \leq 1\end{cases}
$$

In summary we have the estimates

$$
\begin{aligned}
|\zeta(t)| \approx|t|, \quad \|(\zeta(t) & +B)^{-1} \| \\
& \leq 2\left\|(-i t+B)^{-1}\right\| \quad \text { and }\left\|(\zeta(t)-A)^{-1}\right\| \leq C|\Re \zeta(t)|^{-1} .
\end{aligned}
$$

Therefore

$$
|\zeta(t)|\left\|(\zeta(t)+B)^{-1}\right\|\left\|(\zeta(t)-A)^{-1}\right\| \leq \begin{cases}C|t|\left\|(-i t+B)^{-1}\right\|^{2} & |t| \geq 1 \\ C\left\|(-i t+B)^{-1}\right\|^{2} & |t| \leq 1\end{cases}
$$

Figure 2 is a visualization of the components in the setup of our proof.

By elementary contour integral estimates we have the conditions of Theorem 7.2, i.e., (7.23), (7.24), (7.25) and (7.26). The result follows by Theorem 7.2(ii).

(i) If $A$ is invertible we can modify the above contour by joining $\zeta(-1)$ to $\zeta(1)$ by a suitable contour contained in $\{z: \Re z<0\}$, so the resulting contour $\Gamma$ is acceptable. A similar appeal to Theorem 7.2 gives the result.

(ii) is similar, except that we use a contour $\Gamma_{\nu}$ for some $\pi / 2<v<\pi-\phi$.

Theorem 7.4 Suppose $A$ is a sectorial operator such that $-A$ is the generator of a bounded group. Suppose B is an invertible closed operator which commutes with A. Assume further that $\operatorname{Sp}(B) \cap i \mathbb{R}=\emptyset$. Then each of the following conditions implies that the equation $A x+B x=y$ admits a unique mild solution:

(i) A is invertible and

$$
\int_{-\infty}^{\infty}\left\|(i t+B)^{-1}\right\|^{2} d t<\infty
$$




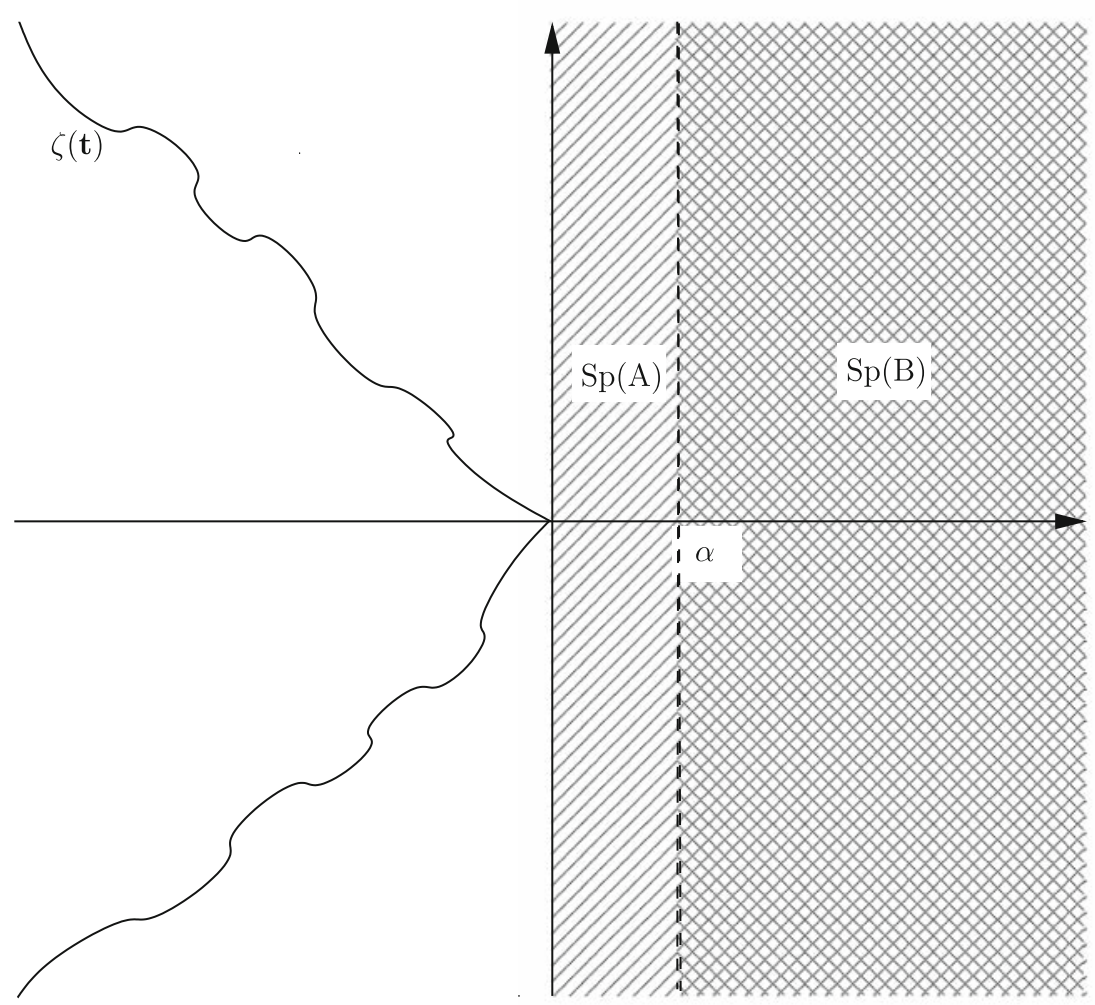

Fig. 2 This figure illustrates Theorem 7.3

(ii) A has an absolute functional calculus and for some $\phi<\pi / 2$ we have $\operatorname{Sp}(B) \subset$ $\Sigma_{\phi} \cup\left(-\Sigma_{\phi}\right)$ and

$$
\sup _{\lambda \notin \Sigma_{\phi} \cup\left(-\Sigma_{\phi}\right)}\left\|(\lambda+B)^{-1}\right\|<\infty .
$$

(iii) A has an absolute functional calculus and

$$
\sup _{-\infty<t<\infty}|t|^{\frac{1}{2}}\left\|(i t+B)^{-1}\right\|<\infty .
$$

Proof As in the previous theorem we first note that the conditions of Proposition 7.1 (i) are satisfied as soon as we have $\sup _{-\infty<t<\infty}\left\|(i t+B)^{-1}\right\|<\infty$. In cases (ii) and (iii) this is automatic. In case (i), we have $\lim _{t \rightarrow \infty}\left\|(i t+B)^{-1}\right\|=0$. For if $s=\frac{1}{2}\left\|(i t+B)^{-1}\right\|$ we have 


$$
\int_{t}^{t+s}\left\|(i \tau+B)^{-1}\right\|^{2} d \tau \geq \frac{1}{2}\left\|(i t+B)^{-1}\right\| .
$$

We will first prove (iii).

In this case, we take $\Gamma$ as the union of two contours. We set

$$
\zeta_{-}(t)= \begin{cases}-i t-\frac{1}{4}\left\|(-i t+B)^{-1}\right\|^{-1} & |t|>1 \\ -i t-\frac{|t|}{4}\left\|(-i t+B)^{-1}\right\|^{-1} & |t| \leq 1 .\end{cases}
$$

and

$$
\zeta_{+}(t)= \begin{cases}i t+\frac{1}{4}\left\|(i t+B)^{-1}\right\|^{-1} & |t|>1 \\ i t+\frac{|t|}{4}\left\|(i t+B)^{-1}\right\|^{-1} & |t| \leq 1 .\end{cases}
$$

These define contours $\Gamma_{1}$ and $\Gamma_{2}$ and it is clear that if $\Gamma=\Gamma_{1} \cup \Gamma_{2}$ we have the conditions of Theorem 7.2.

(i) and (ii) are proved similarly.

Theorem 7.5 Suppose A is a sectorial operator such that $-A$ is the generator of a bounded group with $e^{-2 \pi A}=I$. Suppose $B$ is a closed operator which commutes with $A$. Assume further that $\operatorname{Sp}(B) \cap i \mathbb{Z}=\emptyset$. Then each of the following conditions implies that the equation $A x+B x=y$ admits a unique mild solution:

(i) A is invertible and

$$
\sum_{k \in \mathbb{Z}}\left\|(i k+B)^{-1}\right\|^{2} d t<\infty .
$$

(ii) A has an absolute functional calculus and

$$
\sup _{k \in \mathbb{Z}}|k|^{\frac{1}{2}}\left\|(i k+B)^{-1}\right\|<\infty .
$$

Proof As usual we may verify the conditions of Proposition 7.1 (i) in both cases. We prove (ii). The hypothesis on $B$ implies that $(i t+B)^{-1}$ exists for every $t$ with $|t|>n$ for some natural number $n$. We then define $\Gamma$ to the union of a finite number of contours. First we define a contour $\Gamma_{+-}$by

$$
\zeta(t)=-i t-\frac{1}{2}\left\|(-i t+B)^{-1}\right\|^{-1} \quad t<-n
$$

and $\Gamma_{++}$by

$$
\zeta(t)=i t+\frac{1}{2}\left\|(i t+B)^{-1}\right\|^{-1} \quad t>n
$$




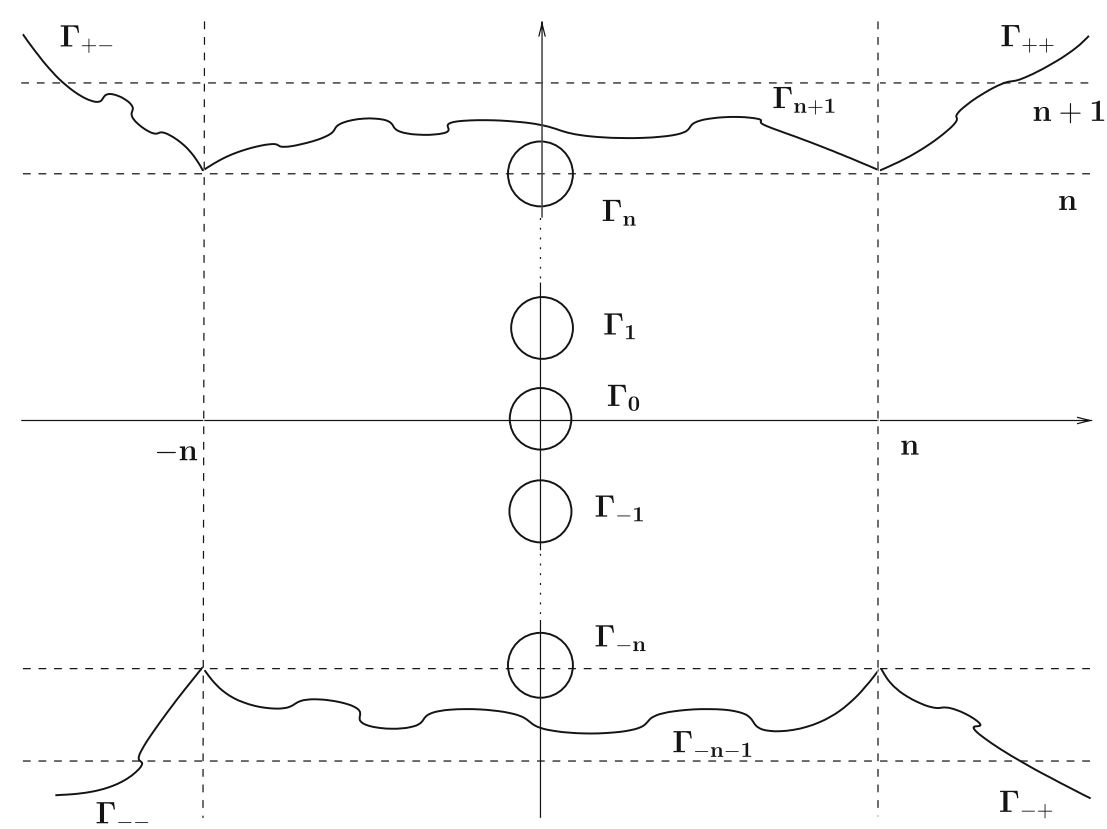

Fig. 3 This figure illustrates Theorem 7.5

We then define $\Gamma_{n+1}$ to be a suitable acceptable contour contained in the resolvent sets of both $-B$ and $A$ passing between $i n$ and $i(n+1)$ to join $\zeta(-n)$ to $\zeta(n)$

Similarly we define $\Gamma_{-+}$by

$$
\zeta(t)=i t+\frac{1}{2}\left\|(i t+B)^{-1}\right\|^{-1} \quad t<-n
$$

and $\Gamma_{--}$by

$$
\zeta(t)=-i t-\frac{1}{2}\left\|(i t+B)^{-1}\right\|^{-1} \quad t>n
$$

and then $\Gamma_{-n-1}$ to be a suitable acceptable contour contained in the resolvent sets of both $-B$ and $A$ and passing between $-i n$ and $-i(n+1)$ to join $\zeta(-n)$ to $\zeta(n)$.

Then let $\Gamma_{k}$ for $|k| \leq n$ be a sufficiently small circle around the point $i k$ contained in the resolvent sets of $A$ and $-B$. If we let $\Gamma$ be the union of all these contours (see figure 3 ) it is clear that the hypotheses of Theorem 7.2 hold and the result follows.

The proof of (i) is similar.

In the each of the preceding Theorems 7.3, 7.4 and 7.5 it is possible to also discuss well-posedness by the same techniques, using the last part of Theorem 7.2. We obtain:

Theorem 7.6 Suppose A is a sectorial operator with an absolute functional calculus such that $-A$ is the generator of a bounded group. Suppose B is a closed operator which commutes with $A$. Then each of the following conditions implies that the equation $A x+B x=y$ is well-posed. 
(i) $\operatorname{Sp}(B) \cap i \mathbb{R}=\varnothing$ and

$$
\sup _{-\infty<t<\infty} t\left\|(i t+B)^{-1}\right\|<\infty
$$

(ii) $e^{2 \pi A}=I, \operatorname{Sp}(B) \cap i \mathbb{Z}=\emptyset$ and

$$
\sup _{k \in \mathbb{Z}}|k|\left\|(i k+B)^{-1}\right\|<\infty
$$

Proposition 7.7 If $A$ generates a bounded group then $-A^{2}$ is sectorial with $\omega\left(-A^{2}\right)=0$ and satisfies an estimate:

$$
\left\|\left(\lambda+A^{2}\right)^{-1}\right\| \leq C|\Im \lambda|^{-1} \Re \lambda>0 .
$$

If further $A$ has an $(a, b)$-absolute functional calculus for some $a, b>0$ then $-A^{2}$ has an $\left(a^{\prime}, b^{\prime}\right)$-absolute functional calculus for $a^{\prime}, b^{\prime}>0$.

Proof If $A$ generates a bounded group then for $\lambda \notin[0, \infty)$ we have

$$
\left(\lambda+A^{2}\right)^{-1}=\frac{1}{2 i \mu}\left((i \mu+A)^{-1}+(i \mu-A)^{-1}\right)
$$

where $\mu^{2}=\lambda$. Sectoriality follows easily and

$$
\left\|\left(\lambda+A^{2}\right)^{-1}\right\| \leq \frac{1}{|2 i \mu|}\left(\left\|(i \mu+A)^{-1}\right\|+\left\|(i \mu-A)^{-1}\right\|\right) \leq C|\mu|^{-1}|\Im \mu|^{-1} .
$$

Note that

$$
|\Im \mu| \geq \frac{1}{2}|\Im \lambda||\mu|^{-1} \quad \Im \lambda>0 .
$$

If $A$ has an $(a, b)$-absolute functional calculus then for a large enough integer $m$, $X$ is a strict real interpolation space for $\left(\mathcal{D}\left(A^{-2 m}\right), \mathcal{D}\left(A^{2 m}\right)\right)$ which then implies that $-A^{2}$ also an $\left(a^{\prime}, b^{\prime}\right)$-absolute functional calculus.

In view of this it is easy to modify our approach to give the following:

Theorem 7.8 Suppose A is a sectorial operator with an absolute functional calculus such that $-A$ is the generator of a bounded group. Suppose A has an $(a, b)$-absolute functional calculus for some $a, b>0$. Suppose $B$ is a closed operator which commutes with $A$. Assume further that $\operatorname{Sp}(B) \cap[0, \infty)=\emptyset$. Suppose

$$
\sup _{t \geq 0} t^{\frac{1}{2}}\left\|(t+B)^{-1}\right\|<\infty .
$$

Then the equation $-A^{2} x+B x=y$ has a unique mild solution for every $y \in X$. 


\section{Applications}

Let us now discuss some related results of Arendt et al. [2]. The authors consider a closed operator $B$ on a Banach space $X$ and a boundary value problem with periodic boundary conditions

$$
u^{\prime}(t)+B u(t)=f(t) \quad u(0)=u(2 \pi)
$$

in the space $\mathcal{C}^{\alpha}(\mathbb{T} ; X)$ of $\alpha$-Hölder continuous $X$-valued functions on the circle (which we identify as the $2 \pi$-periodic functions on $\mathbb{R})$. We denote by $\hat{u}(k)$ the Fourier coefficients

$$
\hat{u}(k)=\frac{1}{2 \pi} \int_{0}^{2 \pi} u(t) e^{-i k t}
$$

Consider the subspace of $\mathcal{C}(\mathbb{T} ; X)$ denoted by $\mathcal{C}_{0}(\mathbb{T} ; X)$ of all functions of means zero. Then, restricted to this space, the differentiation operator $A f=f^{\prime}$ with domain $\mathcal{C}_{0}^{1}(\mathbb{T} ; X)$ of all $C^{1}$-functions of mean zero is sectorial and generates a bounded $2 \pi$-periodic group of translations. In this case $\mathcal{D}(A)=\operatorname{Dom}(A)=\mathcal{C}_{0}^{1}(\mathbb{T} ; X)$.

Now, for $0<\alpha<1$, we consider the inner $(\alpha, \infty)$-interpolation spaces, denoted by $\left(\mathcal{C}_{0}(\mathbb{T} ; X), \mathcal{C}_{0}^{1}(\mathbb{T} ; X)\right)_{(\alpha, \infty)}$, corresponding to the admissible function space

$$
E=\left\{f: \sup _{t>0} t^{-\alpha}|f(t)|<\infty, \lim _{t \rightarrow 0} t^{-\alpha}|f(t)|=\lim _{t \rightarrow \infty} t^{-\alpha}|f(t)|=0\right\} .
$$

This space is the little Hölder space of all mean zero functions $\mathcal{C}_{0}^{\alpha, 0}(\mathbb{T} ; X)$ such that

$$
\sup _{t>s} \frac{\|u(t)-u(s)\|}{|t-s|^{\alpha}}=\|f\|_{\alpha}<\infty
$$

and

$$
\lim _{|t-s| \rightarrow 0} \frac{\|u(t)-u(s)\|}{|t-s|^{\alpha}}=0
$$

See, for example, [21] for the non-periodic case. The following result follows from Corollary 5.7 .

Proposition 8.1 A has a (1,1)-absolute functional calculus on $\mathcal{C}_{0}^{\alpha, 0}(\mathbb{T} ; X)$.

Now if $B$ is a closed operator on $X$ then $B$ induces a closed operator $\tilde{B}$ on $\mathcal{C}_{0}^{\alpha, 0}(\mathbb{T} ; X)$ defined by

$$
(\tilde{B} u)(s)=B u(s) \quad u(s) \in \operatorname{Dom}(B), \quad 0 \leq s \leq 2 \pi .
$$


Now $A+\tilde{B}$ is invertible if and only if the operator

$$
u \rightarrow \sum_{k \in \mathbb{Z}}(i k+B)^{-1} \hat{u}(k) e^{i k t}
$$

extends to a bounded operator on $C_{0}^{\alpha, 0}(\mathbb{T} ; X)$. By an elementary smoothing argument this is equivalent to the boundedness of the same operator on $\mathcal{C}^{\alpha}(\mathbb{T} ; X)$. In view of the discussion of [2] we obtain from Theorem 7.5:

Theorem 8.2 Suppose $0<\alpha<1$. Suppose B is a closed operator on $X$ such that $\operatorname{Sp}(B) \cap i \mathbb{Z}=\emptyset$. Suppose

$$
\sup _{k \in \mathbb{Z}}|k|^{\frac{1}{2}}\left\|(i k+B)^{-1}\right\|<\infty .
$$

Then the equation

$$
u^{\prime}(t)+B u(t)=x(t) \quad u(0)=u(2 \pi)
$$

has a unique mild solution $u \in \mathcal{C}^{\alpha}(\mathbb{T} ; X)$ for every $x \in \mathcal{C}^{\alpha}(\mathbb{T} ; X)$.

Here a mild solution is interpreted in the sense described in [2]. In [2] the same result is obtained with the exponent $1 / 2$ replaced by $2 / 3$, using Fourier multiplier methods. However, for spaces $X$ of nontrivial type they also obtain the same exponent $1 / 2$.

If we consider the same problem in $\mathcal{C}(\mathbb{T} ; X)$, then a similar argument gives the following (in this case the differentiation operator no longer has an absolute functional calculus on $\left.\mathcal{C}_{0}(\mathbb{T} ; X)\right)$ :

Theorem 8.3 Suppose B is a closed operator on $X$ such that $\operatorname{Sp}(B) \cap i \mathbb{Z}=\emptyset$. Suppose

$$
\sum_{k \in \mathbb{Z}}\left\|(i k+B)^{-1}\right\|^{2}<\infty .
$$

Then the equation

$$
u^{\prime}(t)+B u(t)=x(t) \quad u(0)=u(2 \pi)
$$

has a unique mild solution $u \in \mathcal{C}(\mathbb{T} ; X)$ for every $x \in \mathcal{C}(\mathbb{T} ; X)$.

Notice that this condition is weaker than $\left\|(i k+B)^{-1}\right\|=O\left(k^{-\frac{2}{3}}\right)$.

We can also consider second-order equations of the type

$$
-u^{\prime \prime}(t)+B u(t)=x(t) \quad u(0)=u(2 \pi), \quad u^{\prime}(0)=u^{\prime}(2 \pi)
$$

and derive similar results using Theorem 7.8.

The preceding discussion can easily be extended to the non-periodic case. In this case we treat the space $\mathcal{C}_{0}(\mathbb{R})$ of continuous functions vanishes at infinity and we 
obtain that the differentiation operator has an absolute functional calculus on the little Hölder spaces $\mathcal{C}_{0}^{\alpha}(\mathbb{R} ; X)$ of Hölder continuous functions $u: \mathbb{R} \rightarrow X$ vanishing at infinity and such that

$$
\lim _{|t-s| \rightarrow 0} \frac{\|u(t)-u(s)\|}{|t-s|^{\alpha}}=\lim _{|t-s| \rightarrow \infty} \frac{\|u(t)-u(s)\|}{|t-s|^{\alpha}}=0 .
$$

Let us conclude by mentioning another family of examples where our results apply. Let $X$ be an arbitrary Banach space and $N \in \mathbb{N}$. Let $\Delta$ denote the Laplacian $-\sum_{j=1}^{m} \partial^{2} / \partial x_{j}^{2}$. Then $-\Delta$ (as the generator of the heat semigroup) defines a sectorial operator on $L_{q}\left(\mathbb{R}^{N} ; X\right)$ for $1 \leq q<\infty$.

If we let $A=I-\Delta$ then the spaces $\mathcal{D}\left(A^{m}\right)$ for $m \geq 1$ are the Sobolev spaces $W_{q}^{2 m}\left(\mathbb{R}^{N} ; X\right)$. Thus for $1 \leq q, r, s<\infty$

$$
\left(L_{q}\left(\mathbb{R}^{N} ; X\right), W_{q}^{2 m}\left(\mathbb{R}^{N} ; X\right)\right)_{s / 2 m, r}=B_{q, r}^{s}\left(\mathbb{R}^{N} ; X\right)
$$

is a Besov space [1]. It follows that $I-\Delta$ has an absolute functional calculus on the Besov space $B_{q, r}^{s}\left(\mathbb{R}^{N} ; X\right)$ by Theorem 5.5 ; this may also be seen directly from the definitions of these spaces [16]. (If we replace $I-\Delta$ by $-\Delta$ we have that $-\Delta$ has an absolute functional calculus on the corresponding homogeneous Besov space). Fourier multiplier results on Besov spaces are discussed by Amann [1] and Girardi and Weis [16], where it is shown that in contrast to the standard $L_{p}$-cases one does not need R-boundedness assumptions in the hypotheses of these results. These results do not follow from our results but do reflect the fact that $I-\Delta$ has an absolute functional calculus on these spaces.

Acknowledgments The authors acknowledge support from NSF grant DMS-0244515.

Open Access This article is distributed under the terms of the Creative Commons Attribution Noncommercial License which permits any noncommercial use, distribution, and reproduction in any medium, provided the original author(s) and source are credited.

\section{References}

1. Amann, H.: Operator-valued Fourier multipliers, vector-valued Besov spaces, and applications. Math. Nachr. 186, 5-56 (1997)

2. Arendt, W., Batty, C., Bu, S.: Fourier multipliers for Hölder continuous functions and maximal regularity. Studia Math. 160, 23-51 (2004)

3. Auscher, P., McIntosh, A., Nahmod, A.: Holomorphic functional calculi of operators, quadratic estimates and interpolation. Indiana Univ. Math. J. 46, 375-403 (1997)

4. Berens, H., Butzer, P.L.: Approximation theorems for semi-group operators in intermediate spaces. Bull. Amer. Math. Soc. 70, 689-692 (1964)

5. Bourgain, J.: New Banach space properties of the disc algebra and $H^{\infty}$. Acta Math. 152, 1-48 (1984)

6. Bourgain, J., Davis, W.J.: Martingale transforms and complex uniform convexity. Trans. Amer. Math. Soc. 294, 501-515 (1986)

7. Brudny̆ , Y.A., Krugljak, N.Y.: Functors of real interpolation, Russian. Dokl. Akad. Nauk SSSR 256, 14-17 (1981) 
8. Brudnyı̆, Y.A., Krugljak, N.Y.: Interpolation functors and interpolation spaces, vol. I, North-Holland Mathematical Library, 47. Translated from the Russian by Natalie Wadhwa; With a preface by Jaak Peetre, pp. xvi+718. North-Holland, Amsterdam, (1991)

9. Cowling, M., Doust, I., McIntosh, A., Yagi, A.: Banach space operators with a bounded $H^{\infty}$ functional calculus. J. Austral. Math. Soc. Ser. A 60, 51-89 (1996)

10. Cwikel, M.: $K$-divisibility of the $K$-functional and Calderón couples. Ark. Mat. 22, 39-62 (1984)

11. Cwikel, M.: The $K$-divisibility constant for couples of Banach lattices. J. Approx. Theory 124, 124$136(2003)$

12. Cwikel, M., Jawerth, B., Milman, M.: On the fundamental lemma of interpolation theory. J. Approx. Theory 60, 70-82 (1990)

13. Da Prato, G., Grisvard, P.: Equations d'évolution abstraites non linéaires de type parabolique, French, with English summary. Ann. Mat. Pura Appl. (4) 120, 329-396 (1979)

14. Dore, G.: $H^{\infty}$ functional calculus in real interpolation spaces. Studia Math. 137, 161-167 (1999)

15. Dore, G.: $H^{\infty}$ functional calculus in real interpolation spaces. II. Studia Math. 145, 75-83 (2001)

16. Girardi, M., Weis, L.: Operator-valued Fourier multiplier theorems on Besov spaces. Math. Nachr. 251, 34-51 (2003)

17. Kalton, N.J., Kucherenko, T.: Sectorial operators and interpolation theory, interpolation theory and applications, Contemp. Math., 445, pp. 111-119. American Mathematical Society, Providence, RI, (2007)

18. Kalton, N.J., Weis, L.: The $H^{\infty}$-calculus and sums of closed operators. Math. Ann. 321, 319-345 (2001)

19. Lancien, F., Lancien, G., Le Merdy, C.: A joint functional calculus for sectorial operators with commuting resolvents. Proc. Lond. Math. Soc. (3) 77, 387-414 (1998)

20. Lions, J.-L., Peetre, J.: Sur une classe d'espaces d'interpolation, pp. 5-68. Inst. Hautes Études Sci. Publ. Math. (1964) (French)

21. Lunardi, A.: Analytic semigroups and optimal regularity in parabolic problems, progress in nonlinear differential equations and their applications, vol. 16, pp. xviii+424. Birkhäuser Verlag, Basel (1995)

22. McIntosh, A.: Operators which have an $H_{\infty}$ functional calculus, Miniconference on operator theory and partial differential equations (North Ryde, 1986). In: Proc. Centre Math. Anal. Austral. Nat. Univ., vol. 14, pp. 210-231. Austral. Nat. Univ., Canberra (1986)

23. Pisier, G.: Factorization of linear operators and geometry of Banach spaces, CBMS Regional Conference Series in Mathematics, vol. 60, Published for the Conference Board of the Mathematical Sciences, Washington, DC (1986)

24. Triebel, H.: Interpolation theory, function spaces, differential operators, vol. 2, p. 532. Johann Ambrosius Barth, Heidelberg (1995) 